\title{
Tie1 controls angiopoietin function in vascular remodeling and inflammation
}

\author{
Emilia A. Korhonen, ${ }^{1}$ Anita Lampinen, ${ }^{1}$ Hemant Giri, ${ }^{1}$ Andrey Anisimov, ${ }^{1}$ Minah Kim, ${ }^{2}$ Breanna Allen, ${ }^{2}$ Shentong Fang, ${ }^{1}$ \\ Gabriela D’Amico, ${ }^{1}$ Tuomas J. Sipilä, ${ }^{1}$ Marja Lohela, ${ }^{1}$ Tomas Strandin, ${ }^{3}$ Antti Vaheri, ${ }^{3}$ Seppo Ylä-Herttuala, ${ }^{4}$ Gou Young Koh, ${ }^{5,6}$ \\ Donald M. McDonald, ${ }^{2}$ Kari Alitalo, ${ }^{1}$ and Pipsa Saharinen ${ }^{1}$ \\ 'Wihuri Research Institute and Translational Cancer Biology Program, University of Helsinki, Biomedicum Helsinki, Helsinki, Finland. ${ }^{2}$ Department of Anatomy, Cardiovascular Research Institute, and \\ UCSF Helen Diller Family Comprehensive Cancer Center, UCSF, San Francisco, California, USA. ${ }^{3}$ Department of Virology, University of Helsinki, Helsinki, Finland. \\ ${ }^{4}$ A.I. Virtanen Institute for Molecular Sciences, University of Eastern Finland, Kuopio, Finland. ${ }^{5}$ Center for Vascular Research, Institute of Basic Science, and \\ ${ }^{6}$ Graduate School of Medical Science and Engineering, Korea Advanced Institute of Science and Technology (KAIST), Daejeon, Republic of Korea.
}

\begin{abstract}
The angiopoietin/Tie (ANG/Tie) receptor system controls developmental and tumor angiogenesis, inflammatory vascular remodeling, and vessel leakage. ANG1 is a Tie2 agonist that promotes vascular stabilization in inflammation and sepsis, whereas ANG2 is a context-dependent Tie2 agonist or antagonist. A limited understanding of ANG signaling mechanisms and the orphan receptor Tie1 has hindered development of ANG/Tie-targeted therapeutics. Here, we determined that both ANG1 and ANG2 binding to Tie2 increases Tie1-Tie2 interactions in a $\beta_{1}$ integrin-dependent manner and that Tie1 regulates ANG-induced Tie2 trafficking in endothelial cells. Endothelial Tie1 was essential for the agonist activity of ANG1 and autocrine ANG2. Deletion of endothelial Tie1 in mice reduced Tie2 phosphorylation and downstream Akt activation, increased FOX01 nuclear localization and transcriptional activation, and prevented ANG1- and ANG2-induced capillary-to-venous remodeling. However, in acute endotoxemia, the Tie1 ectodomain that is responsible for interaction with Tie2 was rapidly cleaved, ANG1 agonist activity was decreased, and autocrine ANC2 agonist activity was lost, which led to suppression of Tie2 signaling. Tie1 cleavage also occurred in patients with hantavirus infection. These results support a model in which Tie1 directly interacts with Tie2 to promote ANG-induced vascular responses under noninflammatory conditions, whereas in inflammation, Tie1 cleavage contributes to loss of ANG2 agonist activity and vascular stability.
\end{abstract}

\section{Introduction}

The adult vascular system is subject to continuous adaptation to the needs of organ function, mediated by crosstalk between vascular and parenchymal cells $(1,2)$. While angiogenesis research has focused primarily on the mechanisms of vessel sprouting, the remodeling of vascular networks in adult organs has received less attention. Vascular integrity and remodeling are governed by the cooperative interaction of endothelial growth factors and inflammatory cytokines (3).

The endothelial angiopoietin/Tie (ANG/Tie) system regulates angiogenesis during development and tumor growth, contributes to capillary-to-venous remodeling in inflammation, and maintains vascular integrity $(4,5)$. This system comprises the Tie1 and Tie2 (also known as Tek) tyrosine kinase receptors and the Tie2 ligands ANG1, ANG2, and ANG4 (the mouse ortholog is also called ANG3) (6-9). ANG1 is an agonistic paracrine ligand of Tie2 that stimulates Akt-dependent phosphorylation and nuclear

\section{Related Commentary: p. 3188}

Authorship note: E.A. Korhonen and A. Lampinen contributed equally to this work P. Saharinen and K. Alitalo contributed equally to this work.

Conflict of interest: The authors have declared that no conflict of interest exists. Submitted: October 1, 2015; Accepted: June 24, 2016

Reference information: / Clin Invest. 2016;126(9):3495-3510. doi:10.1172/JCI84923. exclusion of the Forkhead box protein O1 (FOXO1) transcription factor (10). FOXO1 inactivation downregulates the expression of genes involved in endothelial destabilization, apoptosis, metabolism, and growth control $(11,12)$. In contrast, ANG2 is an endothelial autocrine ligand that functions as a context-dependent weak agonist or antagonist of Tie2. The functions of ANG3/4 have not been identified, and no ligand has been found for Tie1, which is considered an orphan receptor (5). The function of the Tie1 receptor and the context dependency of ANG2 activity are key unknown factors concerning the mechanisms and functions of the ANG/Tie receptor system of endothelial cells.

ANG1 and Tie2, but not Tie1, are essential for cardiac development, whereas both Tie1 and Tie2 are required for angiogenesis after midgestation $(13,14)$. Tie1-deficient mice die from hemorrhage of the disrupted microvasculature between E13.5 and birth, depending on the genetic background $(15,16)$. These mice also have severe edema from abnormal lymphatic vascular development (16-19). Furthermore, Tie1 is required for sprouting angiogenesis in postnatal development of the retina $(20,21)$. Although Tie1 is downregulated after embryogenesis in some vascular beds, it continues to be expressed in endothelial cells of the heart, lung, and kidney, among other adult organs (22). Tie1 expression is increased at sites of angiogenesis in wound healing, ovarian follicle maturation, and tumors, whereas Tie1 deletion inhibits angiogenesis and tumor growth $(20,22,23)$. 
Tie1 deletion also reduces leukocyte adhesion receptor expression, slows progression of atherosclerosis, and downregulates proinflammatory markers in $A p o e^{-/-}$mice $(24,25)$.

In the adult vasculature, administration of exogenous angiopoietins increases blood vessel size and induces remodeling of capillaries into venules, where leukocyte adhesion receptor expression is high $(26,27)$. This change has some features in common with vascular remodeling after Mycoplasma pulmonis infection of the respiratory tract (28). However, the vascular enlargement induced by angiopoietins in the absence of inflammation is accompanied by resistance to leakage (28). Interestingly, ANG2 blocking antibodies reduce inflammatory vascular remodeling and vascular leakage after M. pulmonis infection and are even more efficacious when combined with TNF- $\alpha$ blockade $(28,29)$. However, the factors that determine whether ANG2 acts as a Tie2 agonist promoting leakage-resistant vessel remodeling or instead as an antagonist that promotes vascular destabilization and leakage remain unknown. In sepsis, significantly elevated ANG2 levels are predictive of poor patient prognosis (30). Reduced Ang2 gene dosage (31), ANG2 blocking antibodies (32), and ectopic ANG1 reduce sepsis-induced vascular leak and lung injury in mice (33). In comparison, mice expressing reduced Tie2 levels are more susceptible to LPS-induced endotoxemia $(34,35)$. ANG2 inhibition also reduces the harmful inflammation associated with cardiac transplant rejection (36) and improves endothelial-pericyte interactions in diabetic retinopathy (37). Some effects of ANG2 associated with pericyte detachment and vascular destabilization involve integrins (38-40). Integrins can also sensitize endothelial cells to low levels of ANG1 (41). Although integrin coupling to pericellular matrix can influence angiopoietin signaling, the underlying mechanisms remain to be determined.

Although Tie1 does not directly bind angiopoietins, it is phosphorylated in endothelial cells after ANG1 stimulation, in a Tie2-dependent manner $(21,42,43)$. Tie1 and Tie 2 can be coimmunoprecipitated from endothelial cells, suggesting that they form a complex (42). Indeed, Tie1 is translocated together with Tie2 to cell-cell contacts where multimeric angiopoietin ligands connect Tie receptors in trans across endothelial cell-cell junctions $(44,45)$. The downstream signaling of Tie1 is poorly understood. Previous studies have shown that ligand stimulation of a CSF-1 receptor/Tie1 receptor chimera results in its autophosphorylation and downstream activation of PI3K and Akt (46). Tie1 deletion in mouse endothelial cells in vivo reduces ANG1-induced phosphorylation of the Tie2 Y1106 residue, indicating that Tie1 is required for ANG1-induced Tie2 activation (20). However, data from other studies suggest that Tie1 inhibits Tie 2 signaling, because Tie2 phosphorylation induced by ANG1 or ANG2 was increased by Tie1 silencing $(43,47,48)$. The role of Tie1 in determining the context-dependent agonist/antagonist action of ANG2 is unknown.

To elucidate the function of Tie1 in angiopoietin signaling during vascular remodeling, we examined Tie1-Tie2 interactions in endothelial cells in vitro and compared effects of ANG1 and ANG2 delivered by adenoviral vectors to WT mice and mice with endothelial cell-specific deletion of Tie1 or inducible endothelial cell-specific overexpression of mouse ANG2. To assess corresponding changes in acute inflammation, we also examined effects of LPS-induced endotoxemia and TNF- $\alpha$ in these models.
We found that angiopoietins promoted a direct interaction of Tie1 and Tie 2 and that this interaction was regulated by integrin $\beta_{1}$. ANG1- and ANG2-induced Tie2 activation and vascular remodeling were reduced or absent in mice where Tie1 was deleted in vascular endothelial cells. Tie1 deletion also attenuated ANG1induced Akt activation and nuclear exclusion of FOXO1. We found that Tie1 was suppressed via ectodomain cleavage during acute inflammation and that this was associated with reduced agonistic activity of ANG2 and decreased Tie 2 signaling. These results indicate that the agonist action of ANG2 is attenuated in inflammation and that Tie 1 is an essential component of the angiopoietin signaling system that has potential for therapeutic targeting in disease.

\section{Results}

Angiopoietins induce direct interaction of Tie1 and Tie2. To investigate the dynamics of the Tie receptors in angiopoietin signaling, we transfected HUVECs with retroviral vectors expressing full-length (FL) Tie1 and Tie2, tagged on the C terminus with mCherry and GFP, respectively. Stimulation of FL-Tie2-GFP and FL-Tie1-mCherry expressing endothelial cells with COMP-Ang1 (CAng1) (49) or with recombinant human ANG2 induced colocalization of Tie1 and Tie 2 in endothelial cell-cell junctions (Figure $1 \mathrm{~A}$ and Supplemental Video 1; supplemental material available online with this article; doi:10.1172/JCI84923DS1) $(44,45,50)$. To investigate potential interactions of Tie1 and Tie2 receptors, we used fluorescence (Förster) resonance energy transfer (FRET), which reports energy transfer between molecules located within $10 \mathrm{~nm}$ of each other. Considering the optimal length of the receptor cytoplasmic domains for FRET (51), we replaced the intracellular domains of Tie1 and Tie2 with the V5-tag and GFP, respectively (Figure 1B). This did not affect angiopoietin-induced receptor translocation to endothelial cell junctions (Figure 1C) $(44,45)$. Tie1- and Tie2-expressing endothelial cells were stimulated with angiopoietins and fixed, and Tie1 was detected using the Cy3-coupled anti-V5 antibody, according to a previously published method for FRET/ fluorescence lifetime imaging microscopy (FLIM) (52, 53). FRET acceptor (Cy3) photobleaching increased the GFP emission intensity, indicating that Tie1 and Tie2 directly interact in unstimulated cells (FRET efficiency 15.0\% $\pm 6.4 \%$ ) (Figure 1, C and E, and Supplemental Figure 1A). Interestingly, the interaction was increased at endothelial cell junctions when the cells were stimulated with CAng1 $(32.1 \% \pm 5.4 \%)$, ANG1 $(30.4 \% \pm 2.7 \%)$, or ANG2 $(29.2 \%$ $\pm 6.8 \%$ ) (Figure $1 \mathrm{E}$ and Supplemental Figure 1A). In contrast, no interaction was detected between Tie1-V5 and a membraneanchored GFP, which was used as a control (FRET efficiency $3.3 \%$ $\pm 0.67 \%$, Supplemental Figure 1B). Furthermore, using frequency domain FLIM microscopy, we observed significantly shorter GFP fluorescence lifetime values in Tie2-GFP- and Tie1-V5-expressing cells after angiopoietin stimulation (CAng1 $1.68 \pm 0.09$ ns, ANG1 $1.64 \pm 0.07 \mathrm{~ns}$, and ANG2 $1.65 \pm 0.06 \mathrm{~ns})$ than in unstimulated control cells $(1.89 \pm 0.07 \mathrm{~ns})$, indicating that angiopoietins increase the interaction of Tie1 and Tie2 (Figure 1, D and F). Again, the strongest interaction was detected at cell-cell junctions. The endothelial adherens junction protein VE-cadherin, expressed as a V5-tagged control protein, did not decrease the GFP lifetime, indicating that despite their localization in cell-cell junctions, Tie2-GFP and VE-cadherin-V5 did not interact (GFP lifetime control $2.0 \pm 0.1 \mathrm{~ns}$ 

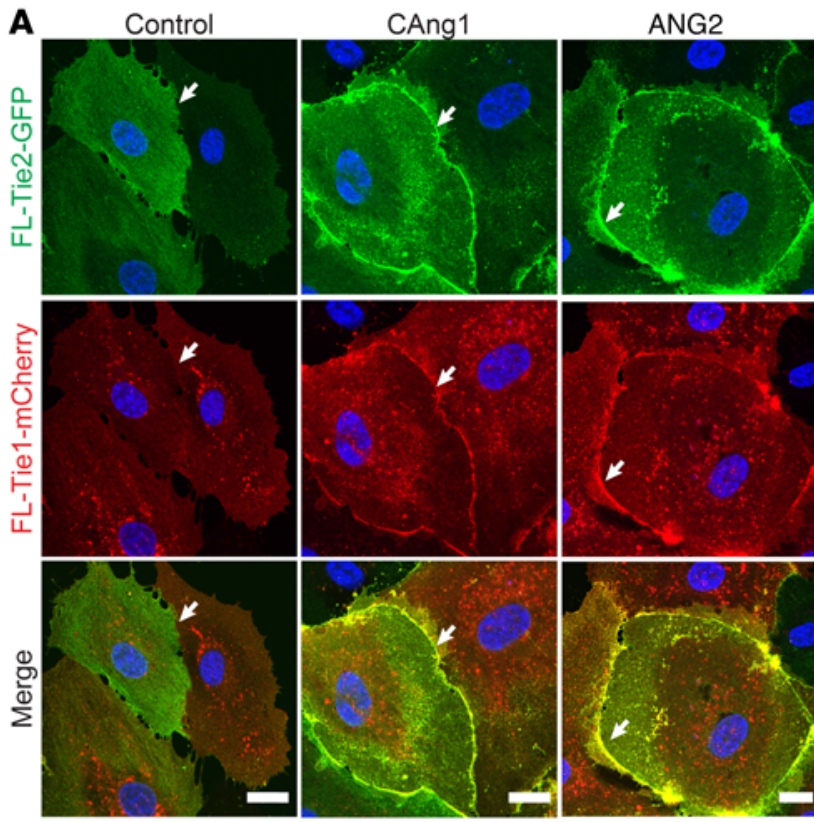

B
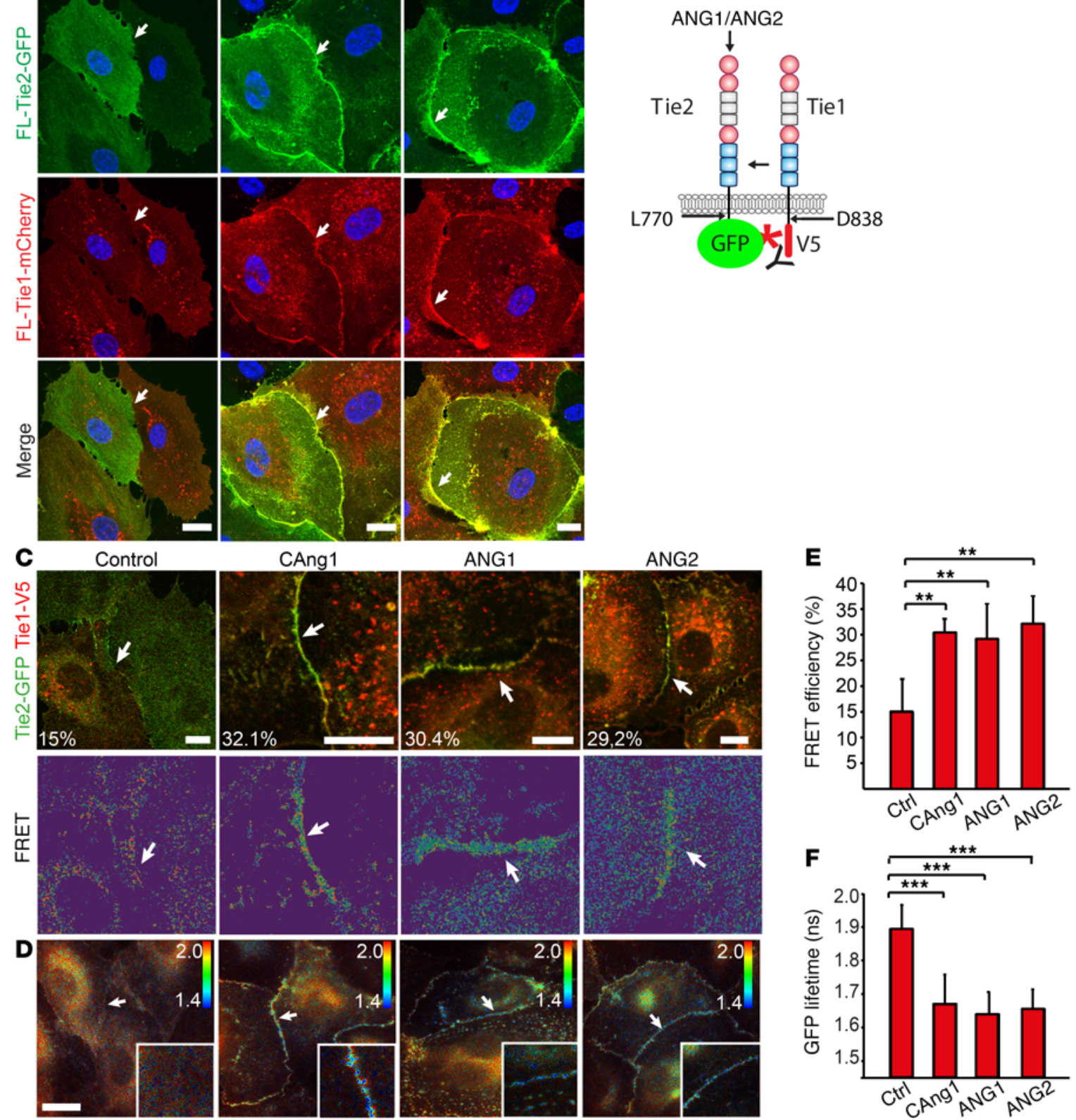

Figure 1. Fluorescence energy transfer between Tie1 and Tie2 after angiopoietin stimulation of endothelial cells. (A) HUVECs were transduced with retroviral vectors encoding FL Tie2-GFP and Tie1-mCherry, stimulated with CAng1, ANG2, or left untreated as a control. Nuclear DAPI stain is shown in blue. (B) Schematic view of the V5- and GFP-tagged Tie1 and Tie2 proteins used for FRET and FLIM. (C) HUVECs were transduced with retroviral vectors encoding the proteins shown in B. Representative confocal images of cells treated with CAng1, ANG1, and ANG2 (upper panel). FRET efficiency percentage was calculated from the ROI after acceptor photobleaching (lower panel). (D) Representative FLIM images (spatial distribution of lifetime value) of HUVECs transduced as in C. Decreased GFP lifetime is indicated by a color change from red to blue. (E) Quantification of FRET efficiency percentage of HUVECs in C from a representative experiment $(n=3) . n($ ROI $)=5$ for unstimulated control, $n($ ROI $)=14$ for CAng1-stimulated, $n($ ROI $)=15$ for ANG1-stimulated, and $n($ ROI) $=14$ for ANC2-stimulated cells. (F) Quantification of GFP lifetimes (ns) of HUVECs in $\mathbf{D}$ from a representative experiment $(n=2) . n($ ROI $)=19$ for unstimulated control, $n(\mathrm{ROI})=42$ for CAng1-stimulated, $n(\mathrm{ROI})=26$ for ANG1-stimulated, and $n(\mathrm{ROI})=33$ for ANC2-stimulated cells. ${ }^{* *} P<0.01 ;{ }^{* * *} P<0.001$, Welch's unequal variances $t$ test, followed by Bonferroni's post hoc test. Error bars indicate SD. Scale bars: $20 \mu \mathrm{m}$. Arrows indicate cell-cell junctions.

vs. CAng1 $1.95 \pm 0.09 \mathrm{~ns}, \mathrm{ANG1} 1.9 \pm 0.09 \mathrm{~ns}, \mathrm{ANG} 21.88 \pm 0.07 \mathrm{~ns})$ (Supplemental Figure 1, C and D). These results indicate that Tie1 is coupled to angiopoietin signaling through direct interaction with Tie2 and that both ANG1 and ANG2 can increase this interaction.

Activation of Tie1 and Tie2 in heteromeric complexes depends on $\beta_{1}$ integrin. Plating on fibronectin can sensitize endothelial cells to ANG1 signaling via $\alpha_{5} \beta_{1}$ integrin-mediated cell adhesion (41). To determine whether $\beta_{1}$ integrin influences the Tie1-Tie2 interaction, we used shRNA lentivirus-mediated gene silencing. Indeed, the interaction was significantly less in $\beta_{1}$ integrin $\left(\operatorname{sh} \beta_{1}\right)$ silenced cells after CAng1 stimulation than in scramble (shScr) silenced controls when measured using FLIM based on time-correlated single photon counting (TCSPC) microscopy (CAng1-stimulated shScr $1.67 \pm 0.09$ ns vs. CAng1-stimulated $\left.\operatorname{sh} \beta_{1} 1.73 \pm 0.09 \mathrm{~ns}\right)$ 
A

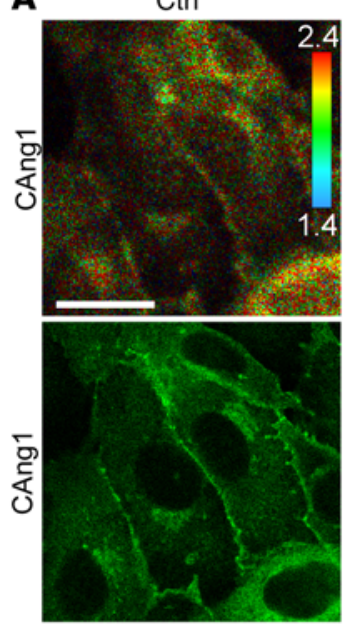

Tie2-GFP

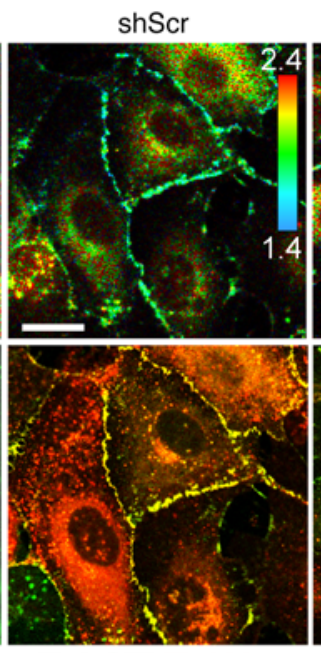

Tie2-GFP Tie1-V5

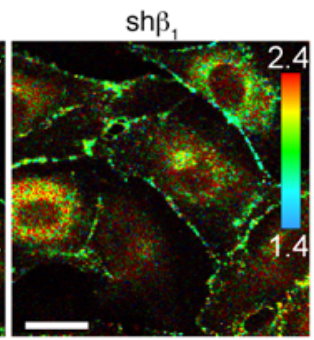

B

C

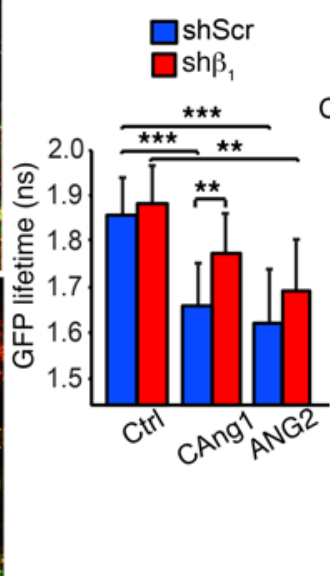

sh Scr $\alpha_{5}$ Scr $\alpha_{5}$ CAng1 - - ++

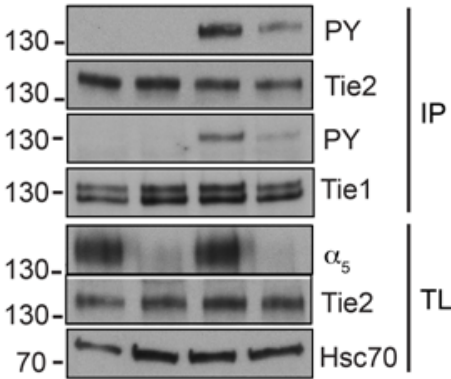

Tie2-GFP Tie1-V5

$\begin{array}{lllllll}D_{\text {Sh }} & \text { Scr } & \beta_{1} & \beta_{3} & \text { Scr } & \beta_{1} & \beta_{3} \\ \text { CAng1 } & - & - & - & + & + & +\end{array}$

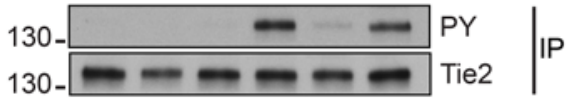

$\begin{array}{ccccccc}\text { sh } & \text { Scr } & \beta_{1} & \beta_{3} & \text { Scr } & \beta_{1} & \beta_{3} \\ \text { VEGF } & - & - & - & + & + & +\end{array}$

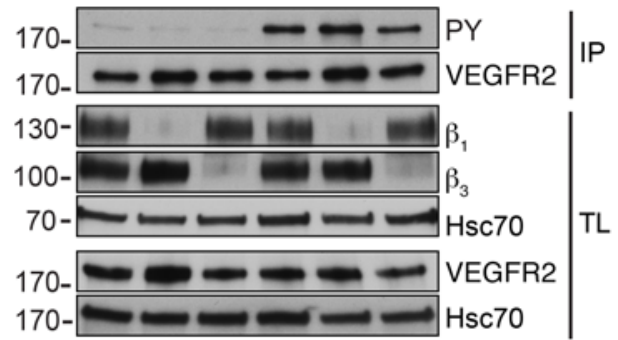

E $\quad$ sh $\operatorname{Scr} \beta_{1}$ Scr $\beta_{1}$

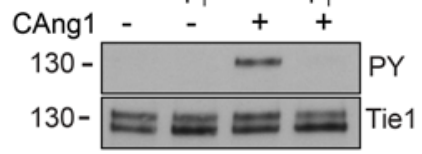

$\mathbf{F}$

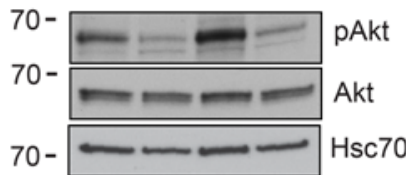

G

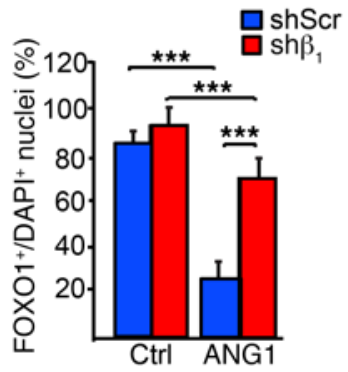

Figure 2. Effect of $\alpha_{s} \beta$ integrin on angiopoietin-induced Tie receptor activation and downstream signaling in endothelial cells. (A) Representative confocal (bottom) and FLIM (top) images of CAng1-stimulated scramble (shScr) or $\beta_{1}$ integrin (sh $\beta_{1}$ ) silenced HUVECs expressing Tie2-CFP alone (left) and with Tie1-V5 (middle and right). Scale bars: $20 \mu \mathrm{m}$. (B) Quantification of GFP lifetimes (ns) $n(\mathrm{ROI})=6$ for unstimulated shScr, $n(\mathrm{ROI})=4$ for unstimulated sh $\beta 1$, $n(\mathrm{ROI})=12$ for CAng1-stimulated shScr, $n(\mathrm{ROI})=16$ for CAng1-stimulated sh $\beta 1, n(\mathrm{ROI})=14$ for ANC2-stimulated shScr, $n(\mathrm{ROI})=13$ for ANG2-stimulated sh $\beta 1$. A representative experiment is shown $(n=2) .{ }^{* *} P<0.01$; ${ }^{* *} P<0.001$, Welch's unequal variances $t$ test, followed by Bonferroni's post hoc test. Error bars indicate SD. (C-F) HUVECs were silenced using shRNA lentivirus for $\operatorname{Scr}(n=10), \alpha_{5}(n=2), \beta_{1}(n=6)$, or $\beta_{3}$ integrin $(n=2)$, stimulated with CAng1 $(n=10)$ or VEGF $(n=1)$, immunoprecipitated (IP) for Tie1, Tie2, or VEGFR2, and analyzed for phosphotyrosine. The membranes were reprobed using Tie1, Tie2, and VEGFR2 antibodies. Total lysates (TL) were analyzed for Hsc70, $\alpha_{5}, \beta_{1}$, or $\beta_{3}$ integrin, Tie1, Tie2, VEGFR2, phospho-Akt $(n=2)$, or Akt, as indicated. (C) Quantification of FOX01-positive nuclei relative to DAPI-positive nuclei (\%) in shScr and sh $\beta 1$ HUVECs stimulated with ANG1. $n$ (cells) $=506$ for unstimulated shScr, $n$ (cells) $=283$ for unstimulated sh $\beta 1$ integrin, $n$ (cells) $=443$ for ANG1-stimulated shScr, $n$ (cells) $=293$ for Ang1-stimulated $\beta$ integrin silenced cells. A representative experiment is shown $(n=2)$. ${ }^{* *} P<0.001$, 1-way ANOVA followed by Tukey's post hoc test.

(Figure 2, A and B, and Supplemental Figure 2A), and FRET acceptor photobleaching (Supplemental Figure 2, B and C). There also tended to be less Tie interaction after ANG2 stimulation of $\beta_{1}$ integrin-silenced cells (Figure 2B, ANG2-stimulated sh $\beta 1$ cells $1.70 \pm 0.11 \mathrm{~ns})$. Tie1/Tie2 translocation to cell-cell junctions was reduced by $\beta_{1}$ integrin silencing in HUVECs when compared with shScr or $\beta_{3}$ integrin silencing (Figure 2A and Supplemental Figure 3). Importantly, silencing of $\alpha_{5}$ integrin (Figure 2C) or $\beta_{1}$ integrin, but not $\beta_{3}$ integrin, reduced the angiopoietin-induced tyrosine phosphorylation of Tie2 and Tie1 (Figure 2, D and E), serine phosphorylation of the downstream Akt kinase (Figure 2F), and nuclear exclusion of the Akt target FOXO1 transcription factor, which is known to control ANG2 expression and endothelial cell functions (54) (Figure $2 \mathrm{G}$ and Supplemental Figure 4A). Inhibition of $\beta_{1}$ integrin binding to fibronectin by blocking antibodies did not affect Tie 2 phosphorylation, and $\beta_{1}$ integrin silencing did not reduce VEGF-induced VEGFR2 phosphorylation (Figure 2D and Supplemental Figure 4B). These results indicate that $\alpha_{5} \beta_{1}$ integrin promotes ANG1-induced formation of heteromeric complexes of Tie1 and Tie2, Tie receptor activation, and downstream signaling. 
A

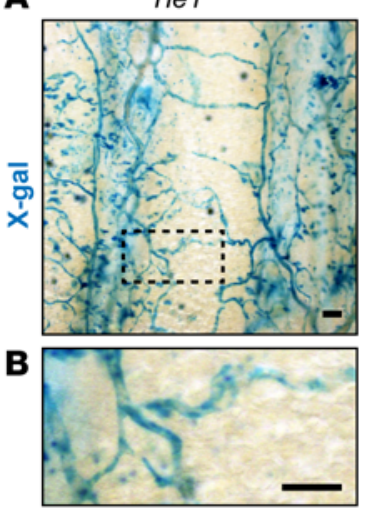

C
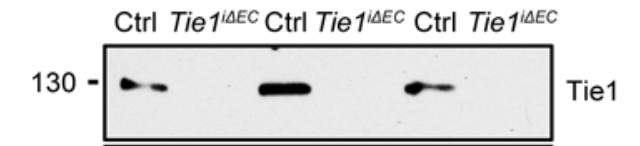

Ad-CAng1: Ad-Ang2:
E

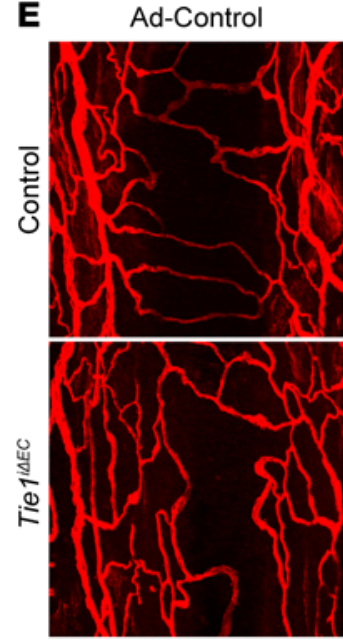

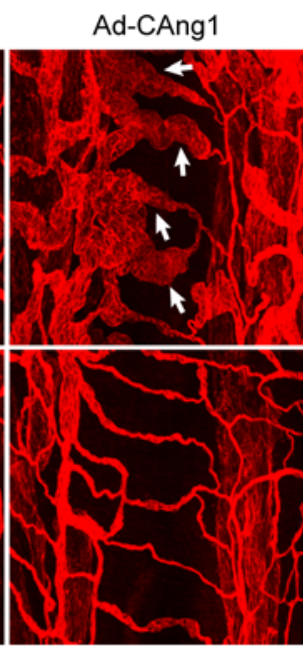

PECAM1

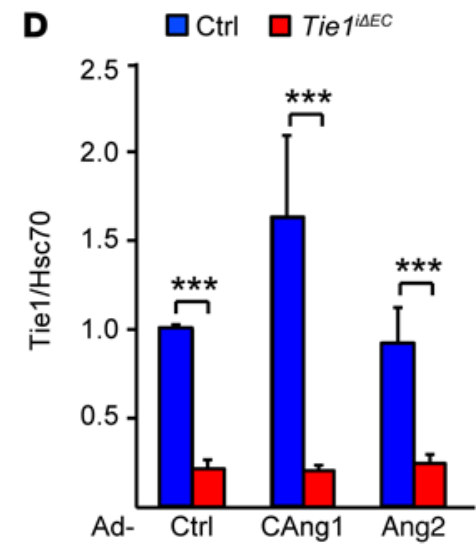

$\mathbf{F}$
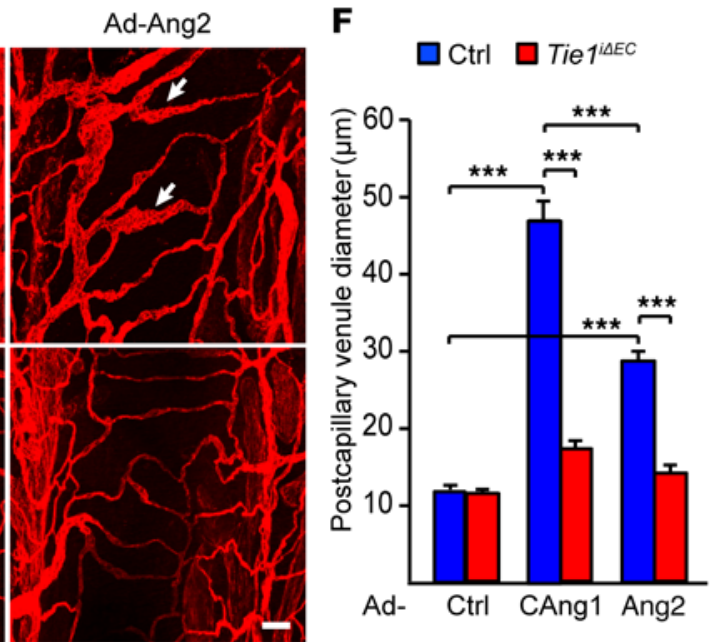

G

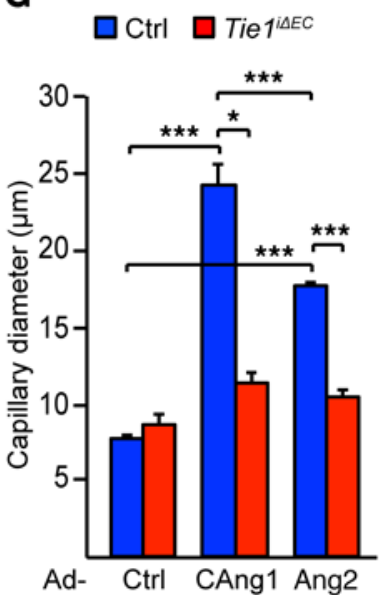

Figure 3. Angiopoietin-induced enlargement of tracheal vessels does not occur in Tie1-deficient mice. (A) Tie1 expression in the tracheal vasculature (X-gal, blue) in a Tie 1tacz/+ mouse. Scale bar: $50 \mu \mathrm{m}$. (B) High magnification of the indicated area in A. Scale bar: $50 \mu \mathrm{m}$. (C) Western blotting of Tie1, CAng1, and ANG2 from lung lysates of control and Tie1-deficient (Tie ${ }^{i \Delta E C}$ ) mice treated with Ad-control, Ad-CAng1, or Ad-Ang2 for 2 weeks. Flag antibody was used to detect angiopoietin expression, and Hsc70 was used as a loading control. (D) Quantification of Tie1 levels relative to loading control from the Western blots in C. Student's $t$ test (control vs. Tie ${ }^{14 E C}$ in each treatment). (E) Tracheal blood vessels visualized by PECAM1 staining. Note the enlarged postcapillary venules and capillaries in Ad-CAng1- and Ad-Ang2-treated control mice (arrows). Scale bar: $50 \mu \mathrm{m}$. (F and G) Diameter measurements for tracheal postcapillary venules $(\mathbf{F})$ and capillaries $(\mu \mathrm{m})(\mathbf{G})$. Error bars indicate SEM. $n=3-11$ (both Pdgfb-iCreER $R^{T 2}$ and $C d h 5[P A C]-C r e E R^{T 2}$ deleters). ${ }^{*} P<0.05$;

${ }^{* * *} P<0.001$, 1-way ANOVA followed by Tukey's post hoc test (F and $\left.\mathbf{G}\right)$.

Tie1 is necessary for vascular remodeling induced by ANG1 or ANG2. To study the role of Tie1 in angiopoietin signaling in vivo, we first investigated Tie1 function in angiopoietin-induced vascular remodeling. Systemic overexpression by adenoviral vectors encoding COMP-Ang1 (Ad-CAng1) and ANG2 (Ad-Ang2) has been shown to induce vascular remodeling in the trachea $(26,27)$. We confirmed Tie1 expression in the tracheal blood vessels of heterozygous Tie ${ }^{\text {lacz/ } /+}$ gene-deleted mice by X-gal staining (Figure 3, A and B) and then deleted both alleles of Tie1 using the endothelial-specific del-

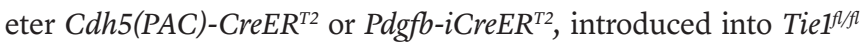
mice and Tie1 ${ }^{f / a c z}$ mice by breeding. Next, angiopoietin expression was induced in Cre-negative control mice and Cre-positive, Tie1deleted (Tie1 ${ }^{\text {iEC }}$ ) littermates by injecting Ad-CAng1 or Ad-Ang2 via the tail vein. Similar levels of CAng1 and ANG2 were detected in the serum (not shown) and lung lysates of adenovirus-transfected control and Tie1-deficient mice (Figure 3, C and D).

Capillaries and postcapillary venules stained for PECAM1 were enlarged in the tracheas of Ad-CAng1- and Ad-Ang2-treat- ed control mice (Figure 3, E-G), in line with previously published results $(26,27)$. Enlargement was greater after Ad-CAng1 than after Ad-Ang2 treatment. However, vascular enlargement was significantly less in tracheas of Tie ${ }^{\text {iSEC }}$ mice given either Ad-CAng1 or Ad-Ang2 (Figure 3, E-G). Vessel size was normal after treatment with control adenovirus, as it was in untreated Tie ${ }^{i \mathrm{AEC}}$ mice. These findings indicate that Tie1 was necessary for enlargement of tracheal blood vessels induced either by Ad-CAng1 or Ad-Ang2.

Tie1 is required for angiopoietin-induced expression of venous markers and endothelial cell proliferation. ANG1-induced capillary enlargement is known to be associated with expression of venous markers involved in leukocyte trafficking in inflammation (27). The venous markers EPHB4 and P-selectin were present in tracheal venules of untreated mice and were stronger in remodeled capillaries of Ad-CAng1- and Ad-Ang2-treated control mice but not in Tie1-deleted mice (Figure 4, A-D). This was not accompanied by significantly increased leukocyte recruitment into the tracheas or peripheral blood of control or Tie1-deleted mice (Supplemental Figure 5, A-D). 
A
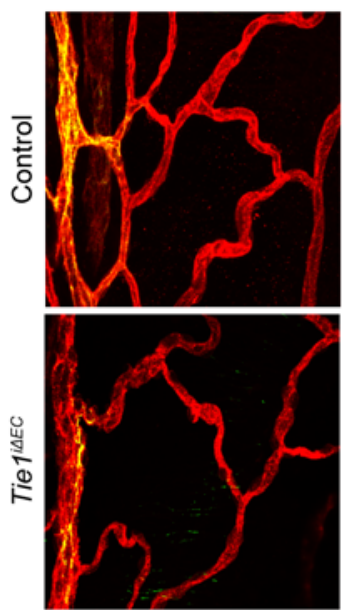

Ad-Control
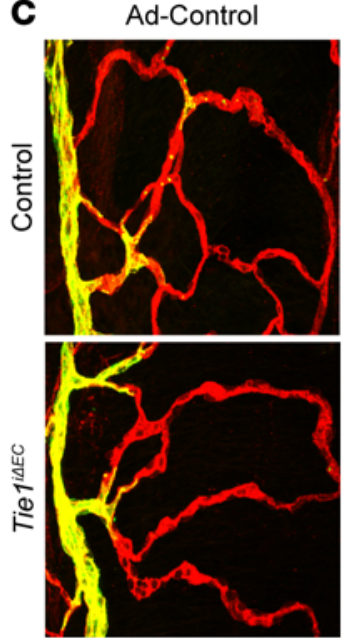

Ad-CAng1

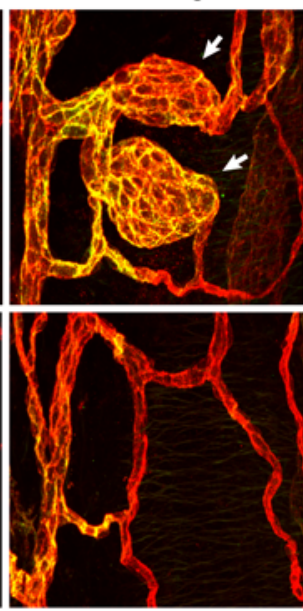

PECAM1 EPHB4
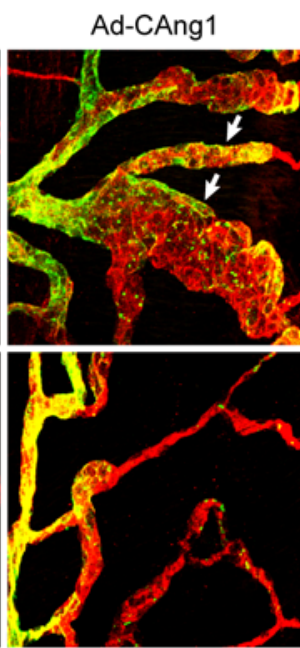

PECAM1 P-selectin

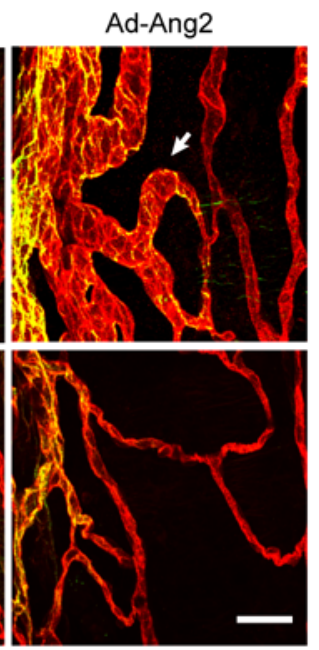

Ad-Ang2
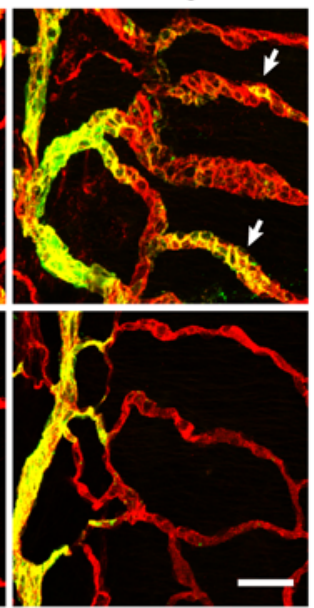

B

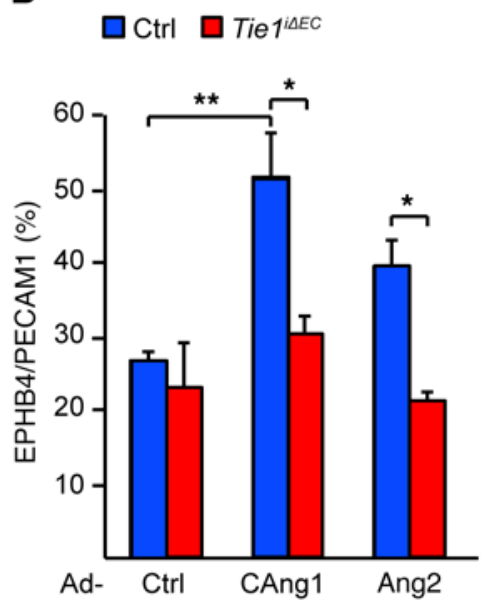

D

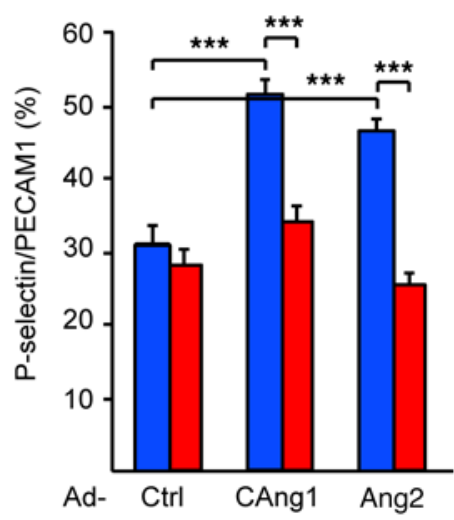

Figure 4. Angiopoietins do not increase EPHB4 and P-selectin expression in Tie1-deleted mice. (A) PECAM1 (red) and EPHB4 (green) staining in tracheal blood vessels of control and Tie ${ }^{\text {iSEC }}$ mice treated with Ad-control, Ad-CAng1, or Ad-Ang2 for 2 weeks. (B) Quantification of EPHB4 area relative to PECAM1 area (\%). $n=3-4$ (Pdgfb-iCreER ${ }^{\text {T2 }}$ deleter). (C) PECAM1 (red) and P-selectin (green) staining in tracheal blood vessels. Note the upregulated EPHB4 and P-selectin expression in the enlarged vessels of Ad-CAng1- and Ad-Ang2-treated control mice (arrows). (D) Quantification of P-selectin. $n=6-11$ (both Pdgfb-iCreER $R^{T 2}$ and Cdh5[PAC]-CreER ${ }^{T 2}$ deleters). Scale bars: $50 \mu \mathrm{m}$. Error bars indicate SEM. ${ }^{*} P<0.05 ;{ }^{* *} P<0.01 ;{ }^{* * *} P<0.001,1-$ way ANOVA followed by Tukey's post hoc test.

ANG1 has been shown to increase endothelial cell proliferation during vascular remodeling (55). Both Ad-CAng1 and Ad-Ang2 increased endothelial cell proliferation in control mice, but had little effect in Tie1-deficient mice, as shown by staining for the cell proliferation marker Ki67 (Figure 5, A and B). Therefore, we silenced Tie1 in HUVECs using shTie1 lentivirus (Figure 5C) and analyzed endothelial cell proliferation using time-lapse imaging and biochemical analysis by the MTT cell proliferation assay. Tie1-silenced HUVECs proliferated less than untreated or shScr-transfected HUVECs (Figure 5, D and E). However, staining for activated caspase-3/7 indicated that apoptosis of Tie1-silenced HUVECs was increased only when the cells were cultured in serum-free conditions (Supplemental Figure 6). Thus, Tie1 silencing in cultured endothelial cells reduced cell proliferation as after in vivo deletion.

Tie1 deficiency impairs ANG1-induced Tie2 and Akt phosphorylation and FOXO1 inactivation. To further investigate the attenuated responses of Tie1-deficient mice to Ad-CAng1 or Ad-Ang2, we ana- lyzed Tie2 phosphorylation. Tie2 phosphorylation in lung lysates of control mice was greater at 2 days after Ad-CAng1, but not after Ad-Ang2 (Figure 6A). Tie1 deletion decreased the phosphorylation of Tie2 and Akt after Ad-CAng1 (Figure 6A). FOXO1 staining was restricted to the cytoplasm of control HUVECs stimulated with CAng1, but was in the nucleus of Tie1-silenced HUVECs after CAng1, as in unstimulated control HUVECs (Figure 6, B and C). ANG2 caused FOXO1 nuclear exclusion in only some cells (Figure $6, \mathrm{~B}$ and C). Increased cytoplasmic FOXO1 staining was detected in endothelial cells of tracheal vessels after Ad-CAng1, but this tended to be less in Tie1-deleted mice (Figure 6D and Supplemental Figure 7). Consistent with FOXO1 inactivation, Ad-CAng1 treatment decreased expression of the FOXO1 target genes endothelial cell specific molecule 1 (Esm1) and Ang2 in the tracheas and lungs of control mice, but the decrease was less in Tie1-deleted mice (Figure 6E) (54). Ad-Ang2 increased Tie1 and reduced Esm1 expression, suggesting agonist ANG2 activity in the trachea (Figure 6E). 
A
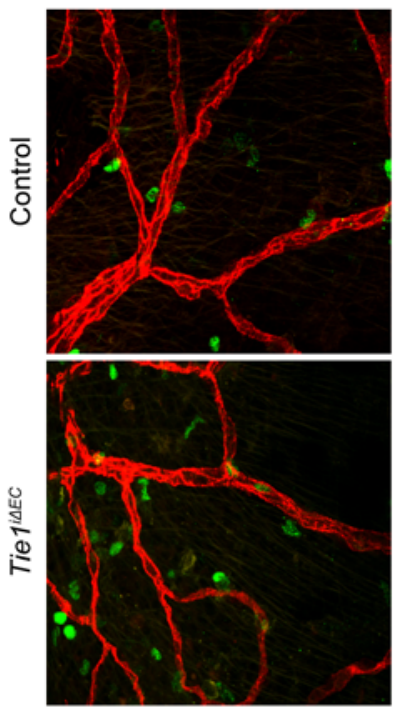

C

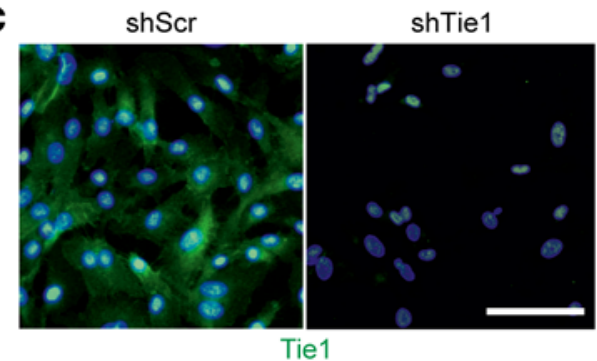

Ad-CAng1
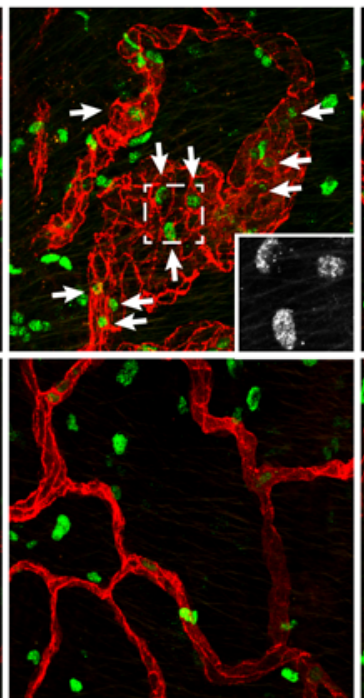

PECAM1 Ki67
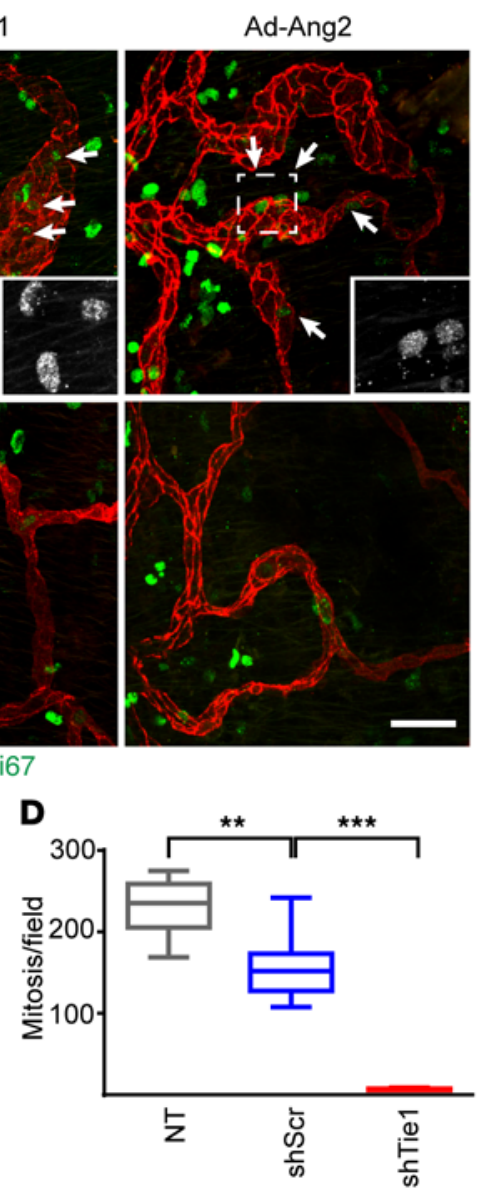

B

$\square$ Ctrl $\square$ Tie1 $1^{\text {ISEC }}$

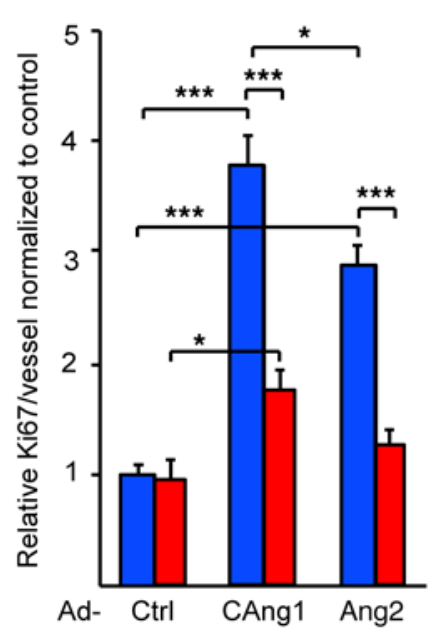

E

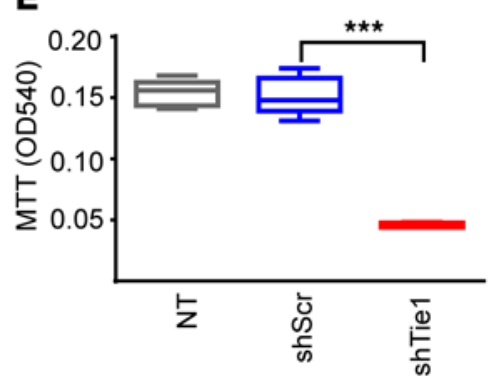

Figure 5. Tie1 deficiency reduces angiopoietin-induced endothelial cell proliferation. (A) PECAM1 (red) and Ki67 (green) staining of tracheal blood vessels of control and Tie ${ }^{\text {LEC }}$ mice treated with Ad-control, Ad-CAng1, or Ad-Ang2 for 2 weeks. Arrows point to Ki67-positive endothelial cells. (B) Quantification of number of Ki67-positive endothelial cells relative to PECAM1 area in the tracheas, normalized to Ad-control-treated control mice. $n=6-9$ (both Pdgfb-iCreER ${ }^{T 2}$ and Cdh5[PAC]-CreER ${ }^{T 2}$ deleters). (C) Tie1 staining of HUVECs transfected with lentivirus encoding Tie1 shRNA (shTie1) or shScr. (D and E) Number of mitotic cell divisions counted from Cell-IQ video microscopy images (D) and amount of live cells as determined by MTT assay (E) in Tie1-silenced, shScr, and nontransfected cells (NT). $n=4$. Scale bars: $50 \mu \mathrm{m}$. Error bars indicate SEM. ${ }^{*} P<0.05$; ${ }^{* *} P<0.01$; ${ }^{* * *} P<0.001,1$-way ANOVA followed by Tukey's post hoc test.

Tie1 is required for ANG2 agonist action on Tie2 under baseline conditions. To mimic the autocrine induction of ANG2, we used a tetracycline-regulated transgenic mouse model expressing a VE-cadherin-tTA (Cdh5-tTA) or Tie1-tTA (used in the FVB genetic background) transgene to overexpress mouse ANG2 in the endothelium (Cdh5-tTA tetO-Ang2 or Tie1-tTA tetO-Ang2 mice, referred to hereafter as $A n g 2^{E C}$ ) (Supplemental Figure 8) (50). We induced ANG2 expression on PO by omitting tetracycline from the drinking water. Analysis of the double-transgenic $A n g 2^{E C}$ mice at 2 to 3 months of age showed enlargement of postcapillary venules and capillaries in the trachea as well as somewhat increased leukocyte counts (Figure 7, A-C, and Supplemental Figure 9, A-D). Ang2 transgene expression for 7 to 11 months in mice of the FVB genetic background led to enlargement of vessels in the trachea and ear skin. The blood vessels in the ear skin were also tortuous and had scattered aneurysm-like enlargements (Supplemental Figure 10, A and B). Quantitative reverse-transcription PCR (qRT-PCR) analysis of the lungs of ANG2 overexpressing mice revealed greater than normal Tie1, but not Tie2, gene expression and less Esm1 expression, consistent with increased Tie2 agonistic activity (Figure 7D).
To investigate the involvement of Tie1 in the agonistic function of ANG2, we introduced the Pdgfb-iCreERT2 deleter and conditional Tie1 alleles into the conditional $A n g 2^{E C}$ mice. ANG2 expression was induced in these mice at PO, and Tie1 was deleted in the adults. Western blotting of lung lysates demonstrated the efficiency of ANG2 induction, which was associated with increased Tie1 levels and loss of Tie1 protein in the gene-deleted mice (Figure 7E). Strikingly, Tie2 phosphorylation promoted by endothelial ANG2 overexpression did not occur in the Tie1-deleted mice (Figure 7F), indicating that Tie1 was necessary for the Tie2 agonistic activity of autocrine ANG2. Similarly, when ANG2 agonist activity was tested in HUVECs ectopically expressing FL-Tie2-GFP, Tie1 silencing reduced Tie2 phosphorylation (Figure $7 \mathrm{G}$ and Supplemental Figure 11A). Accumulation of FL-Tie2-GFP at endothelial cell junctions after ANG2 stimulation was also less in Tie1-silenced cells than in shScr-treated controls (Supplemental Videos 2 and 3; Supplemental Figure 11B). Tie2GFP-positive vesicles were more numerous after ANG1 stimulation of Tie1-silenced HUVECs when compared to shScr-treated or ANG2 stimulated HUVECs (Supplemental Videos 4 and 5; Supplemental Figure 11C, and Supplemental Figure 12A). Consistent with Tie2 internalization in Tie1-silenced HUVECs, Tie2 protein in the lungs 
A

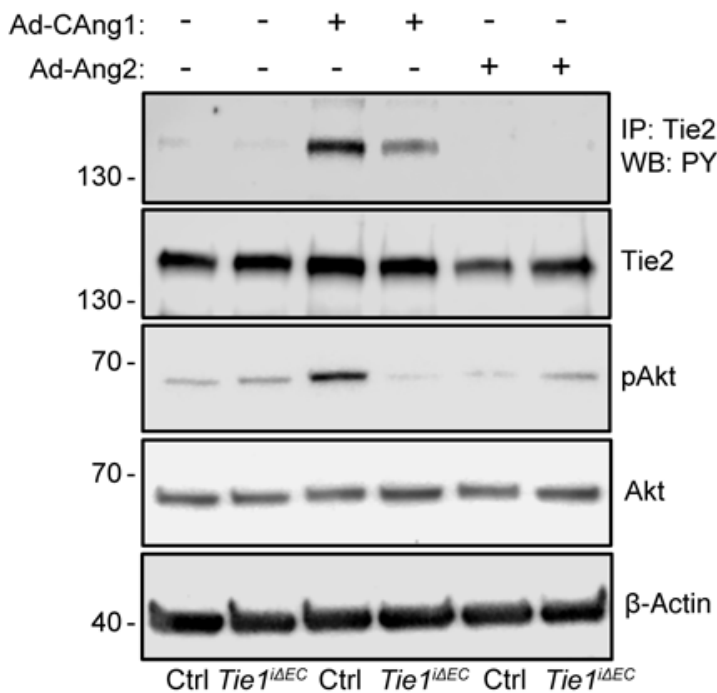

C

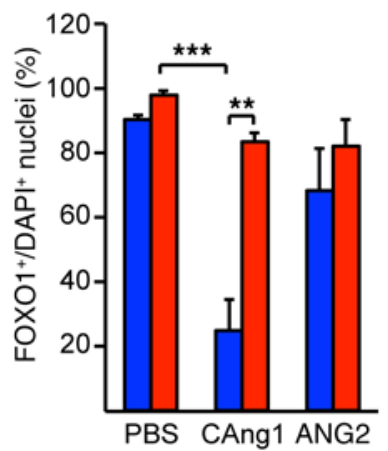

D $\square$ Ctrl $\square T$ Tie $1^{\text {IAEC }}$

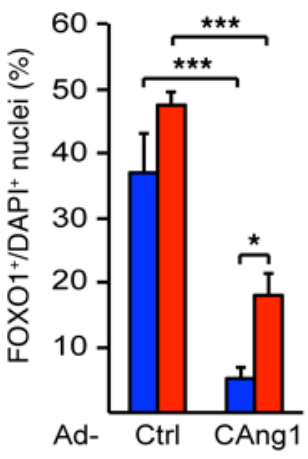

B

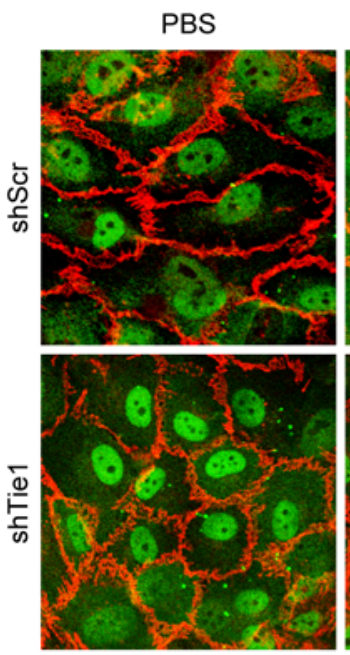

E

Ctrl, Ad-Ctrl Q Tie ${ }^{\triangle \triangle E C}$, Ad-Ctrl

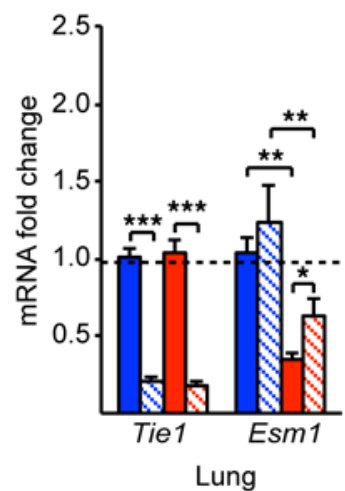

CAng1

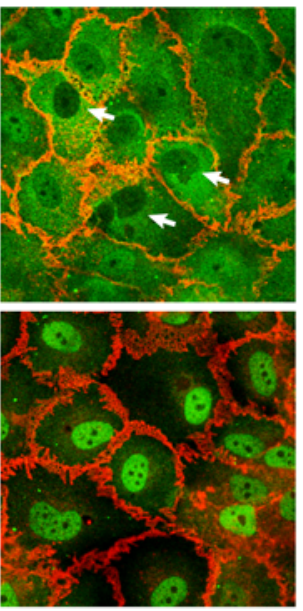

VE-cadherin FOXO1

Ctrl, Ad-CAng1

Q Tie ${ }^{\triangle \triangle E C}$, Ad-CAng1

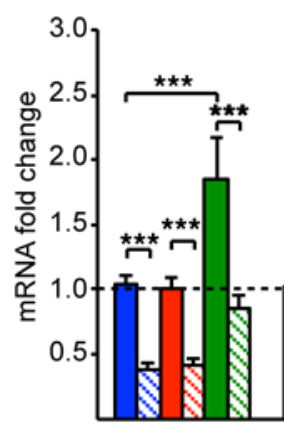

Tie1
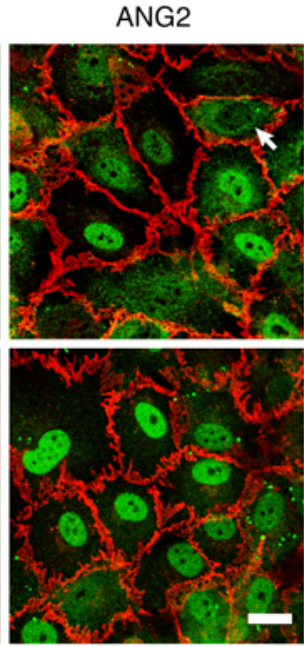

Ctrl, Ad-Ang2

Tie ${ }^{\mathrm{A}}{ }^{\mathrm{A} C}, \mathrm{Ad}-\mathrm{Ang} 2$

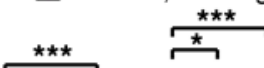

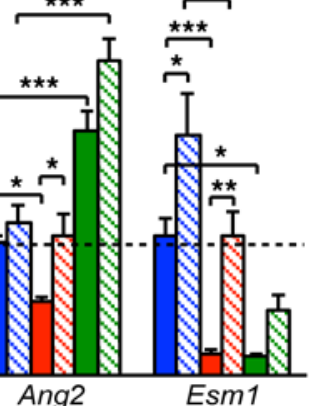

Figure 6. Tie1 deficiency suppresses ANG1-mediated FOX01 inactivation both in vitro and in vivo. (A) Western blotting of phospho-Tie2, total Tie2, phospho-Akt, total Akt, and $\beta$-actin from lung lysates of control and Tie ${ }^{\text {idEEC }}$ mice treated with Ad-control, Ad-CAng1, or Ad-Ang2 for 2 days. Representative Western blots $(n=4-9)$. (B) VE-cadherin (red) and FOX01 (green) staining of shScr- and shTie1-transfected HUVECs stimulated with CAng1 or ANG2. Arrows point to FOXO1-negative nuclei. Scale bar: $20 \mu \mathrm{m}$. (C and D) Quantification of FOXO1-positive nuclei relative to DAPI-positive nuclei (\%) in HUVECs $(n=3)(C)$ and in tracheal blood vessels $(n=4)(D) .(E)$ qRT-PCR analysis of Tie1 and the Foxo1 targets Esm1 and Ang2 mRNA fold changes in the lungs and tracheas. The values are normalized to Pecam1 mRNA expression. $n=4-11$ (both Pdgfb-iCreER ${ }^{T 2}$ and Cdh5[PAC]-CreER ${ }^{T 2}$ deleters). Error bars indicate SEM. ${ }^{*} P<0.05 ;{ }^{* *} P<0.01 ;{ }^{* *} P<0.001$, 1-way ANOVA followed by Tukey's post hoc test (C and $\mathbf{E}$ : trachea) and Fisher's LSD test ( $\mathbf{D}$ and $\mathbf{E}:$ lung).

of Ad-CAng1-treated, Tie1-deleted mice was less than in controls (Supplemental Figure 12B). These results indicate that Ad-CAng1 was a much stronger Tie2 agonist than ANG2 and that the agonist activity of both ANG1 and ANG2 was dependent on Tie1.

LPS promotes rapid Tie1 cleavage in inflammation. Decreased levels of Tie 2 phosphorylation and protein have been observed in inflammatory diseases associated with increased ANG2 expression $(56,57)$. In line with this, Tie2 staining in tracheal blood vessels decreased in a time-dependent manner from 1 to 16 hours after LPS challenge (Figure 8, A-C). Similarly, Tie2 in lung lysates was about $30 \%$ less at 16 hours after LPS, but the leukocyte adhesion protein VCAM1 was greater than the control (Figure 8, D and E, and Supplemental Figure 13A). However, we discovered that Tie1 immunofluorescence was rapidly lost from tracheal blood vessels after LPS challenge (Figure 8, A and C). Western blot analysis using an antibody against the Tie1 ectodomain demon- strated a reduction in the amount of FL Tie1 and the presence of a reduced molecular weight form of Tie1 in lung lysates, representing cleaved Tie1 ectodomain (Figure 8, D and E, and Supplemental Figure 13A). Also, the level of Tie1 ectodomain in serum was greater than in the control as soon as 30 minutes after LPS (Figure 8F). Tie1, Tie2, and Ang1 mRNAs were significantly lower in the lungs of LPS-challenged mice than in controls, but the kinetics were slower than for Tie1 protein (Supplemental Figure 13, B and C).

To determine whether Tie1 cleavage occurred in human inflammatory disease, we then measured soluble Tie1 ectodomain (sTie1) in the serum of patients with acute Puumala hantavirus (PUUV) disease (58). The levels of sTie1 were significantly greater in the affected patients than in healthy controls (Figure 8G).

TNF- $\alpha$ is a key cytokine upregulated in sepsis, many infections, and by systemic administration of LPS (59). Tnf and Il1b mRNA in the lungs and TNF- $\alpha$ protein in the serum were signifi- 
A
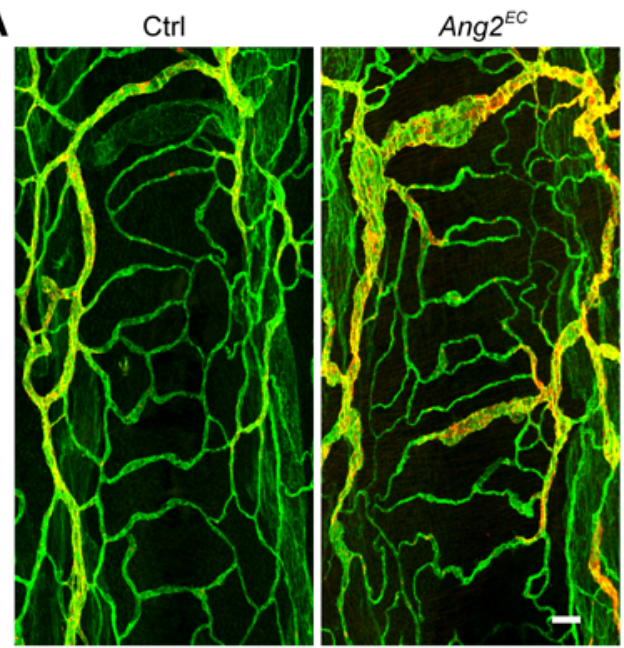

B

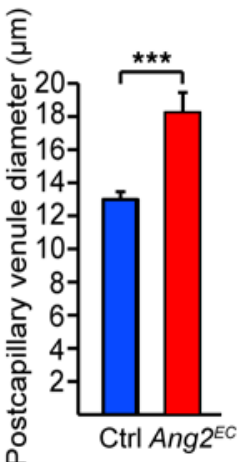

PECAM1 P-selectin

E

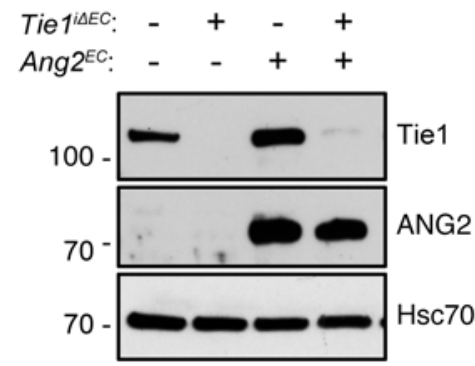

$\mathbf{F}$
C

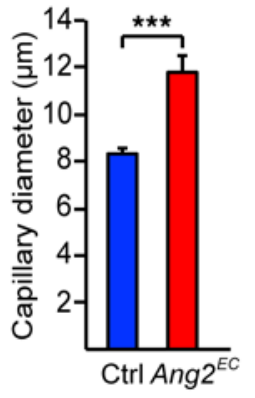

D

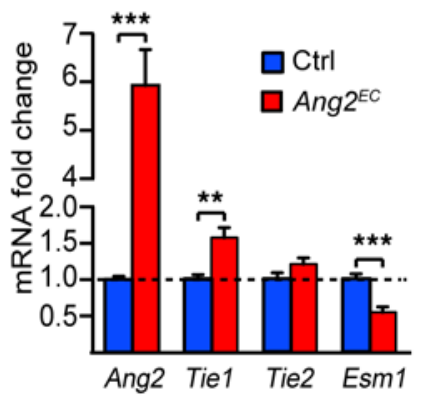

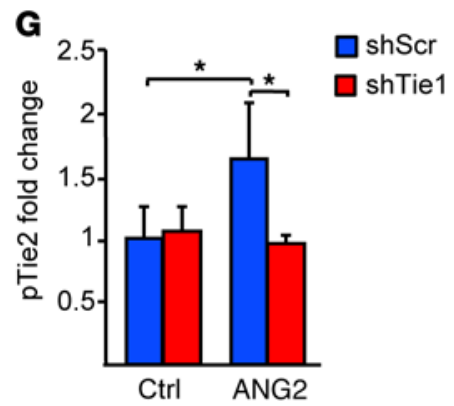

Figure 7. Tie1 deletion inhibits autocrine ANG2 agonist activity in ANG2 transgenic mice. (A) ANG2 overexpression in the vascular endothelium was induced at birth, and PECAM1 (green) and P-selectin (red) were stained to visualize the tracheal blood vessels in 2- to 3-month-old control and ANG2-overexpressing $\left(\right.$ Ang ${ }^{{ }^{E C}}$ ) mice. (B and C) Diameter measurements for tracheal postcapillary venules (B) and capillaries ( $\left.\mu \mathrm{m}\right)$ (C). (D) qRT-PCR analysis of Ang2, Tie1, Tie2, and Esm1 mRNA fold changes in the lungs. The values are normalized to Pecam1 mRNA expression. (E and F) Tie1, ANG2, Hsc70 (E), phospho-Tie2, and Tie2 (F)

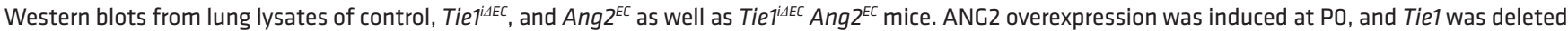
in 6- to 10-week-old mice, which were analyzed 3 weeks after the first dose of tamoxifen. A representative experiment is shown ( $n=5-10)$. Note that ANG2 expression is associated with upregulation of Tie1 mRNA (D) and protein (E). Scale bar: $50 \mu \mathrm{m}$. Error bars indicate SEM. $n=5-10$ (Pdgfb-iCreER ${ }^{T 2}$ deleter). ${ }^{* *} P<0.01$; ${ }^{* *} P<0.001$, Student's $t$ test. (C) Quantification of Tie2 phosphorylation (pTie2/nuclei, 4 microscopic fields/treatment) after ANG2 stimulation in shScr and Tie1-silenced FL-Tie2-GFP-expressing HUVECs. Data of a representative experiment are shown as a ratio to shScr control (representative confocal images are shown in Supplemental Figure 11A, $n=2$ ). Error bars indicate SD. ${ }^{*} P<0.05$, 1-way ANOVA followed by Tukey's post hoc test.

cantly greater at 1 hour after LPS challenge (Supplemental Figure $14, \mathrm{~A}$ and B). As TNF- $\alpha$ is known to induce Tie1 cleavage in vitro (60), we tested its effect on Tie1 cleavage in vivo. Injection of TNF- $\alpha$ into mice resulted in weaker Tie1 staining in the trachea at 1 hour (Supplemental Figure 14C) and greater sTie1 in serum, consistent with TNF- $\alpha$ promotion of Tie1 cleavage (Supplemental Figure 14D). Sequestration of TNF- $\alpha$ by pretreatment with soluble TNF- $\alpha$ receptor prevented the sharp increase in sTie1 in serum after TNF- $\alpha$, but not after LPS (Supplemental Figure 14, D and E), suggesting that additional inflammatory signals are involved in LPS-mediated Tie1 cleavage.

LPS-induced inflammation suppresses ANG2 agonist function. Tie2 phosphorylation was less in the lung lysates and tracheal vessels of LPS-treated mice than in corresponding controls (Figure 9A and Supplemental Figure 15, A and B). The phospho-Akt/ Akt ratio was also less (Figure 8D), which fits with greater nuclear FOXO1 in tracheal vessels of LPS-challenged mice (Supplemental Figure 15, C and D). Staining for ANG2 and von Willebrand factor (vWF) was much weaker in the tracheal vessels at 1 hour after LPS challenge, consistent with release from Weibel-Palade bodies (Supplemental Figure 16, A-C). These rapid changes were followed by an increase of Ang2 mRNA in the lungs and a gradual rise of ANG2 protein in serum (Supplemental Figure 16, D and E). These findings indicate that after LPS, ANG2 was greater in tissue and serum, but did not promote Tie2 phosphorylation and thus did not act as a Tie2 agonist.

To investigate the contribution of Tie1 cleavage on ANG2 agonist activity in inflammation, we measured phospho-Tie2 by Western blotting of lung lysates of $A n g 2^{E C}$ mice. Tie 2 was phosphorylated in the lungs of $A n g 2^{E C}$ mice under baseline conditions, but not after LPS challenge (Figure 9, A and B). Similarly, Tie2 phosphorylation in the lungs of WT mice was greater after Ad-CAng1 and weaker after LPS challenge (Figure 9, C and D). Thus, similar to genetic deletion of Tie1, loss of the Tie2 interacting ectodomain of Tie1 after LPS was accompanied by reduced agonistic effects of angiopoietins on Tie2. Agonist effects of ANG1 were weakened, and those of ANG2 were abolished.

CAng1 inhibits inflammation-induced Tie1 cleavage. ANG1 is known to decrease LPS-induced inflammation and vascular leakage by promoting Tie2 signaling (33). In line with this, we found that Ad-CAng1 injected 2 days before LPS challenge decreased LPS-induced leakage in tracheal vessels, Ang2 mRNA in lungs, 
A
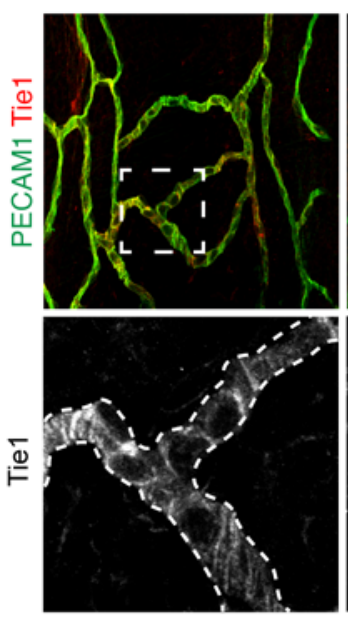

LPS
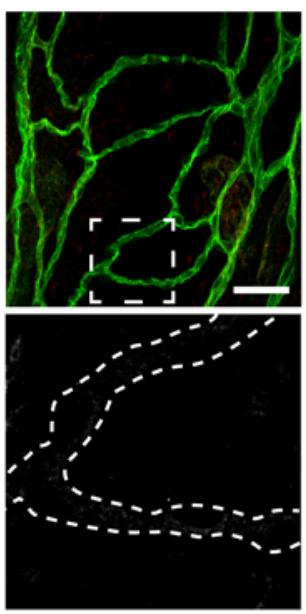

B
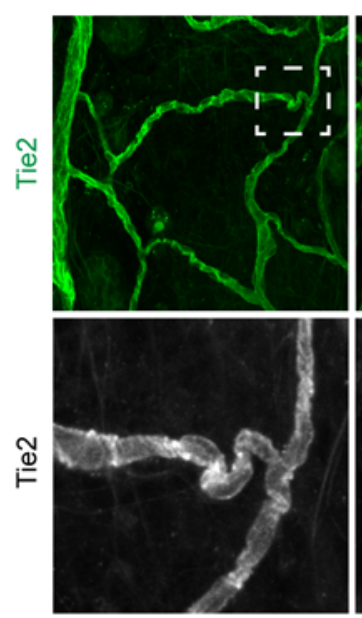

LPS
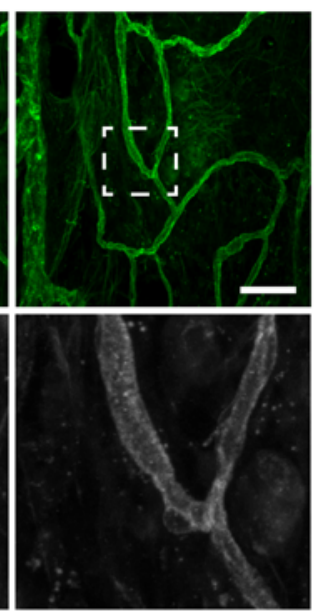

C
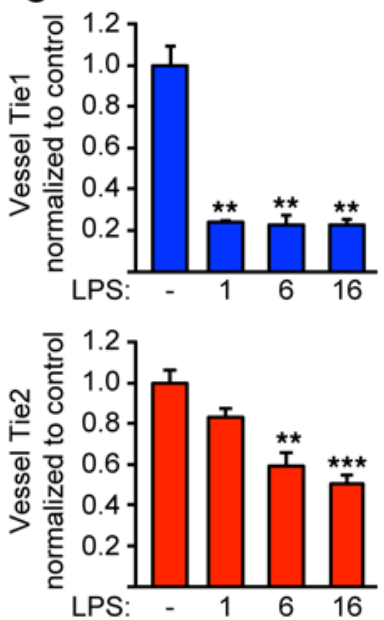

D

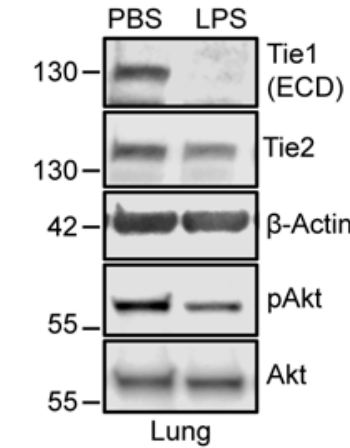

$\mathbf{F}$

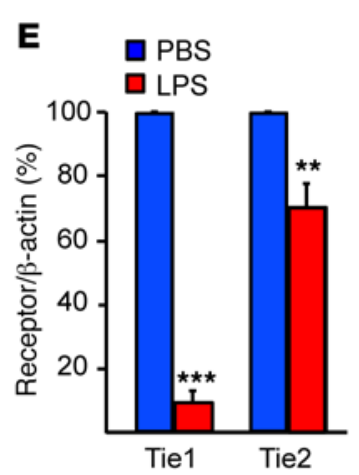

Serum

G

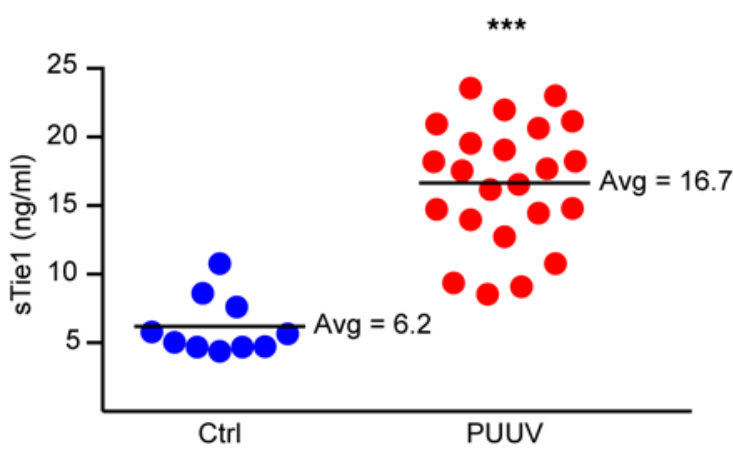
Tie1
(ECD)

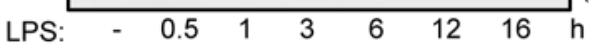

Figure 8. Inflammation induces Tie1 cleavage and subsequent decrease of Tie2. (A) Tie1 expression in tracheal blood vessels of mice treated with LPS for 16 hours, Tie1 (red, white), and PECAM1 (green) staining. (B) Tie2 expression (green, white) in tracheal blood vessels in the same experiment. Scale bars: 50 $\mu \mathrm{m}$. (C) Quantification of Tie1 and Tie2 immunostaining in tracheal vessels of the LPS-treated mice, normalized to PBS-treated mice at the indicated time points (h). $n=3 .{ }^{* *} P<0.01,{ }^{* *} P<0.001,1$-way ANOVA followed by Dunnett's post hoc test. (D) Western blotting of Tie1 extracellular domain (ECD), Tie2, $\beta$-actin, phospho-Akt, and Akt in lung lysates from PBS- and LPS-treated control mice. (E) Quantification of Tie1 and Tie2 protein levels from Western blots in D. $n=4$, Student's $t$ test. (F) Representative Western blot of Tie1 in serum from LPS-treated mice at the indicated time points ( $n=2-6)$. (G) sTie1 protein $(\mathrm{ng} / \mathrm{ml})$ in serum from control $(n=10)$ and PUUV disease patients $(n=23)$. ${ }^{*} P<0.01 ;{ }^{* *} P<0.001$, Student's $t$ test. Error bars indicate SEM.

and ANG2 protein in serum (Supplemental Figure 17, A-D). Tie2 activity was reduced but still present in lung lysates of LPS-challenged mice pretreated with Ad-CAng1 (Figure 9, C and D). Similarly, Tie1 was greater in lungs and tracheal vessels after Ad-CAng1 than after Ad-control (Figure 9, G-I). These findings indicate that ANG1 attenuates LPS-induced loss of Tie1, which fits with the vascular protective effects of ANG1. In contrast, high ANG2 expression in the $A n g 2^{E C}$ mice neither activated Tie2 nor prevented the loss of Tie1 after LPS challenge (Figure 9, A, B, E, and F), which is consistent with ANG2 acting as a Tie2 antagonist under these inflammatory conditions.

\section{Discussion}

Learning how the ANG/Tie system governs vascular stability and remodeling in health and disease has been limited by an incomplete understanding of the contribution of the orphan Tie1 receptor in angiopoietin signaling and the context-dependent function of ANG2. We show here that ANG1 and ANG2 binding to Tie2 increases Tie1-Tie2 interaction in signaling complexes that translocate to cell-cell junctions in a $\beta_{1}$ integrin-dependent manner and that Tie1 regulates normal receptor trafficking. We found that after Tie1 deletion in gene-targeted mice, adenovirally delivered ANG1 or transgenic overexpression of ANG2 induced little or no Tie2 phosphorylation and ANG1 did not induce AktFOXO signaling or suppress FOXO1-regulated gene expression. Furthermore, ANG1- and ANG2-induced endothelial cell proliferation, upregulation of venular markers, and enlargement of capillaries and postcapillary venules typical of vascular remodeling in sustained airway infection did not occur in the Tie1-deleted mice $(27,28,55)$. These findings indicate that loss of Tie1 reduces or prevents the agonistic activity of ANG1 and ANG2. In acute inflammation after LPS challenge, the Tie1 ectodomain was rapidly cleaved. This was followed by reduced Tie 2 phosphorylation, downregulation of Tie2 and ANG1 expression, and upregulation of ANG2. The results suggest that Tie1 interactions with Tie2 promote ANG1- and ANG2-induced Tie2 signaling under base- 
A
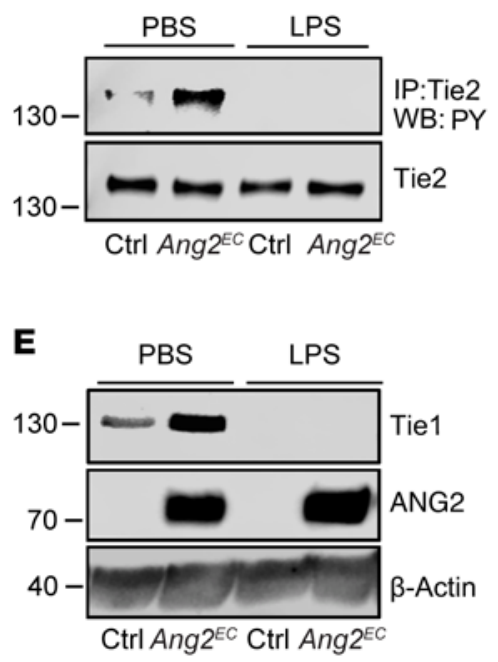

I PBS, Ad-Control
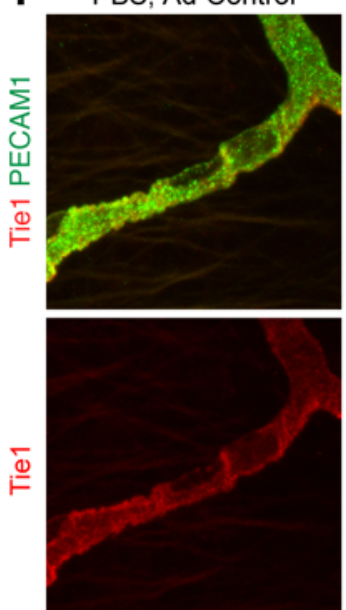

B
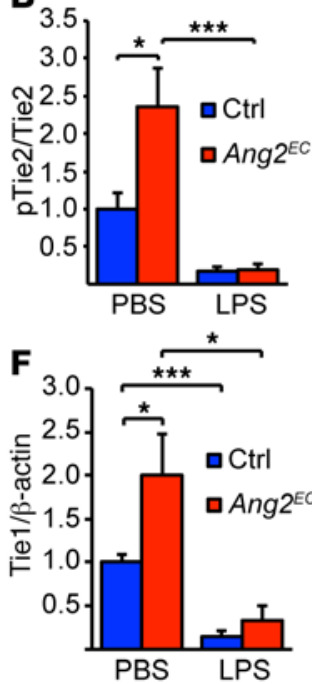

PBS, Ad-CAng1

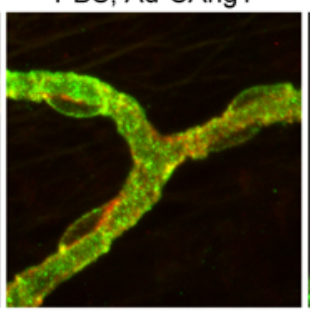

C

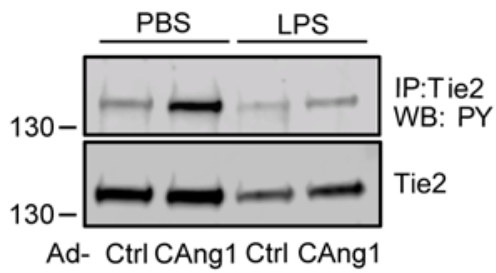

G

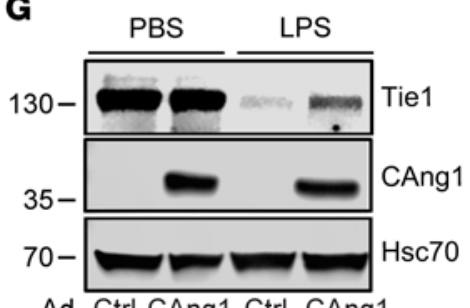

Ad- Ctrl CAng1 Ctrl CAng1
D
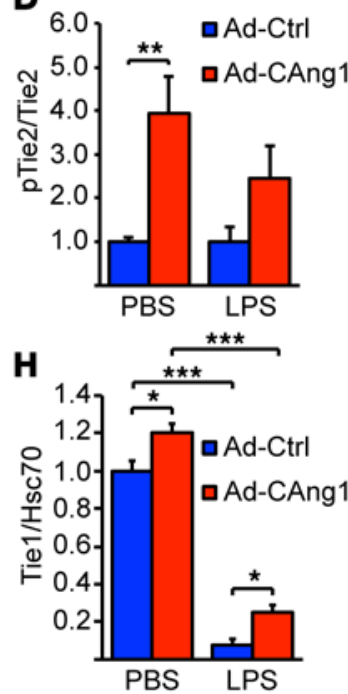

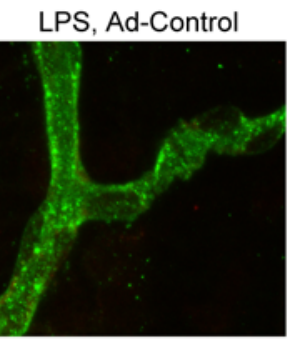

LPS, Ad-CAng1

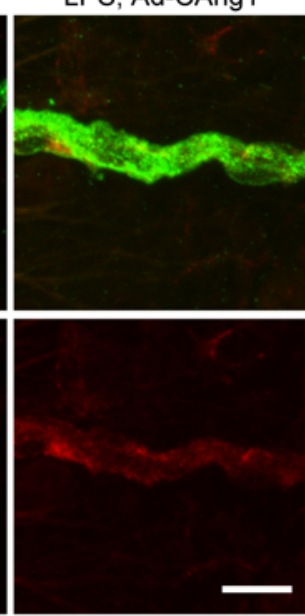

Figure 9. Effect of LPS on Tie1 levels and ANG1- and autocrine ANG2-induced Tie2 phosphorylation. (A) Western blot showing phosphorylation of Tie2 in lung lysates from control and Ang2 $2^{E C}$ mice treated with PBS or LPS. (B) Quantification of phospho-Tie2 relative to total Tie2 from the Western blots in A (C) Western blot showing phosphorylation of Tie2 in lung lysates of Ad-control- and Ad-CAng1-treated mice with or without LPS treatment. (D) Quantification of phospho-Tie2 relative to total Tie2 from the Western blots in $\mathbf{C}$. (E) Western blotting of Tie1, ANC2, and $\beta$-actin in lung lysates from control and Ang $2^{E C}$ mice treated with PBS or LPS. (F) Quantification of Tie1 levels relative to $\beta$-actin from the Western blots in E. (G) Western blotting of Tie1, Flag, and Hsc70 in lung lysates from mice treated with Ad-control and Ad-CAng1 with or without LPS treatment. Flag antibody was used to detect CAng1 expression. (H) Quantification of Tie1 levels relative to Hsc70 from the Western blots in E. Error bars indicate SEM. $n=3-7$. ${ }^{*} P<0.05$; ${ }^{* *} P<0.01$; ${ }^{* * *} P<0.001,1-$ way ANOVA followed by Tukey's post hoc test. (I) Tie1 (red) and PECAM1 (green) staining of tracheal blood vessels of Ad-control- and Ad-CAng1-treated mice with or without LPS treatment. Representative images $(n=8)$. Scale bar: $20 \mu \mathrm{m}$.

line (noninflammatory) conditions and that loss of Tie1 in acute inflammation reduces the agonist action of ANG1, prevents the agonist action of ANG2, and promotes vascular destabilization (Figure 10). Of clinical relevance, sTie1 concentration was found to be abnormally high in serum of patients with acute PUUV disease, suggesting that Tie1 cleavage is involved in vascular destabilization in that condition.

Angiopoietin-induced Tie1-Tie2 interaction and vessel remodeling. Tie1 does not directly bind angiopoietins, but as we and others have reported, Tie1 coimmunoprecipitates in a heteromeric complex with Tie2 (42) and is phosphorylated by ANG1 in a Tie2-dependent manner in vitro $(21,42,43)$. However, the ligand-induced trafficking of the Tie receptors has distinctive features. The activated Tie complex becomes enriched at endothelial cell-cell junctions, where it resists solubilization by nonionic detergents, possibly because it is bound to the actin cytoskeleton or special lipid microdomains (45). Therefore, instead of analyzing the Tie receptor complexes in solution, we chose to probe Tie1-Tie2 interactions using FRET/ FLIM microscopy in intact endothelial cells.

By using TCSPC/FLIM/FRET, which detects short-range $(<10 \mathrm{~nm})$ molecular interactions, we found that Tie1 and Tie2 form molecular complexes that are promoted by angiopoietin stimulation, resulting in ANG1-induced activation of both Tie1 and Tie2. Despite promoting the Tie1-Tie2 interaction, ANG2 induces little or no Tie phosphorylation in endothelial cell cultures unless the Tie receptors are ectopically expressed $(9,42,45,61)$. Similar to Tie1 deletion in $A n g 2^{E C}$ mice, Tie1 silencing in HUVECs reduced ANG2-induced phosphorylation and junctional translocation of 
A

Vascular remodeling in response to ANG1 and ANG2

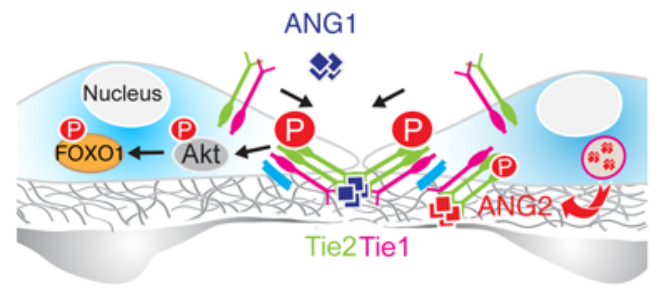

Ang-induced Tie2 phosphorylation

Tie1-Tie2 complex

FOXO1 in cytoplasm

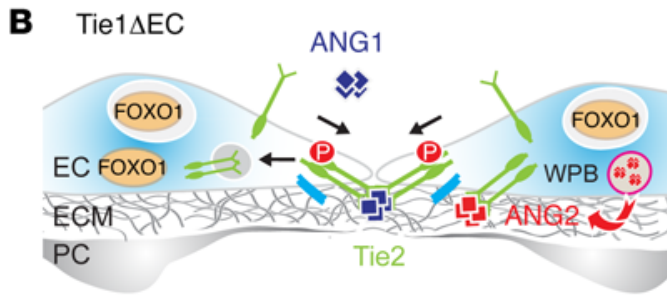

Decreased vascular remodeling Reduced Tie2 phosphorylation Increased Tie2 internalization Less FOXO1 in cytoplasm

\section{Acute inflammation}

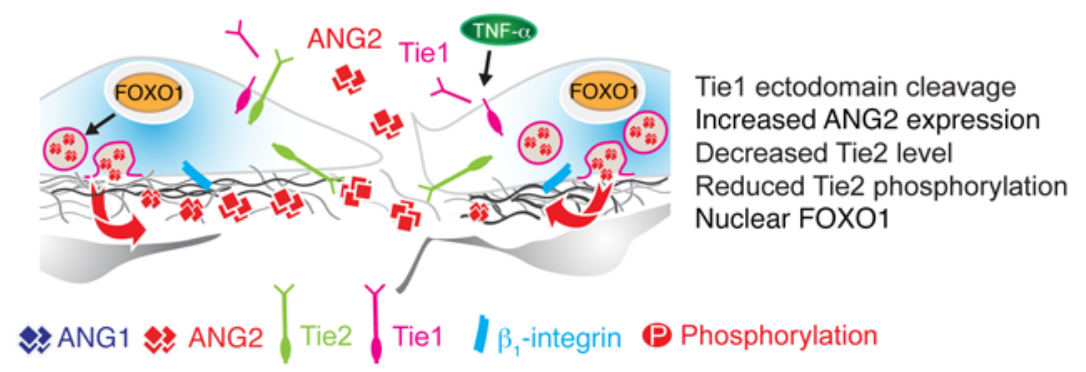

Figure 10. Model of Ang-Tie signaling during vessel remodeling and inflammation. (A and $B$ ) ANG1- and ANG2-induced vascular remodeling requires Tie1, which is recruited to the Tie2 signaling complex at endothelial cell-cell junctions after ligand stimulation in a $\beta_{1}$ integrin-dependent manner. Under noninflammatory conditions, ANG1 activates the Tie2/Akt pathway, leading to FOXO1 nuclear exclusion. Autocrine ANG2 has similar effects as a weak agonist. (C) In LPS-induced acute inflammation, ANC2 is released from Weibel-Palade bodies (WPBs), but Tie1 cleavage inhibits ANG2 agonist activity, leading to FOXO1 nuclear translocation and Ang2 gene transcription via a self-enforcing loop. These rapid changes are followed by decreased phospho-Tie2, Tie2, and ANG1 and impaired integrity of the inflamed vasculature. In these conditions, ANG2 switches from Tie2 agonist to antagonist and via $\beta$ integrin, may further promote vascular destabilization. EC, endothelial cell; PC, pericyte; ECM, extracellular matrix. the ectopically expressed Tie 2 and promoted ANG1-induced Tie2 translocation into vesicular structures, where it colocalized with early endosome antigen 1 (EEA-1) (62). These results challenge the model of Seegar et al. (48), who reported that Tie1 inhibits ANG2 signaling and that ANG1 promotes Tie1 dissociation from Tie2, measured by acceptor photobleaching in U2OS osteosarcoma tumor cells and EA.hy926 somatic endothelial-tumor hybrid cells. In contrast, our results indicate that Tie 1 is required for the agonist activity of ANG1 and ANG2 in vivo and that Tie1-Tie2 interaction increases after angiopoietin stimulation of primary endothelial cells.

We also found that silencing of $\beta_{1}$ integrin, but not $\beta_{3}$ integrin, had effects similar to silencing Tie1, where ANG1-induced activation of Tie1, Tie2, and Akt was reduced and nuclear FOXO1 was increased. These results fit with previous findings demonstrating greater ANG1-Tie2 signaling in endothelial cells adherent to fibronectin matrix via $\alpha_{5} \beta_{1}$ integrin (41). In contrast, ANG2 can activate $\beta_{1}$ integrin directly, independent of Tie2 $(38,40)$, which promotes radial stress fiber formation, endothelial destabilization (40), and pericyte detachment (39).

Our findings provide evidence that Tie1 receptors are directly involved in angiopoietin signal transduction that leads to vascular remodeling, consistent with previous results on the involvement of Tie1, ANG2, Tie1+Tie2, and ANG1 in postnatal development of the retinal vasculature $(20,21,63,64)$. However, our results do not support reports that Tie1 downregulates Tie2 signaling and limits endothelial cell survival $(43,65)$. In contrast, the abnormal vascularity and leakage found in Tie1-null mouse embryos could be explained by reduced ANG1-induced Tie2 activation (15). Adding further complexity, the functions of Tie1 and angiopoietins in blood vessels differ from those in lymphatic vessels and the functions during development differ from those in adult disease processes $(16-19,63,66)$.

Activated Tie 2 promotes downstream signaling via Akt, which phosphorylates FOXO1, leading to its nuclear exclusion and downregulation of FOXO1 target genes $(10,54)$. This is the likely mechanism of endothelial cell proliferation associated with angiopoietin-induced vascular enlargement under noninflammatory conditions. Interestingly, activating mutations of the Tie2 gene increase downstream Akt phosphorylation and promote aberrant vascular remodeling in human venous malformations $(67,68)$. Deletion of Tie1 prevented angiopoietin-induced vascular remodeling, but the contribution of Tie1 to aberrant vascular remodeling of human venous malformations remains to be explored.

Requirement of Tie1 for ANG2 agonist activity in vivo. Our results indicate that ANG2 acts as a Tie2 agonist in $A n g 2^{E C}$ mice and that this activity, as Ad-Ang2-induced vascular remodeling, did not occur in the absence of Tie1. Previous studies using cultured endothelial cells have suggested that ANG2 functions as a weak autocrine agonist and a stabilizing factor of stressed endothelial cells, especially in the absence of ANG1, which normally provides a vascular protective signal that limits angiogenesis, tissue fibrosis, and organ damage $(54,69)$. Ad-Ang2 induced less vascular remodeling than Ad-CAng1, and no significant Tie2 phosphorylation remained at 2 days after Ad-Ang2 administration. Interestingly, decreased Esm1 mRNA expression was found in the tracheas of Ad-Ang2treated mice, suggesting that ANG2 had agonist activity. The reason for the weaker Tie2 phosphorylation in the lungs observed after Ad-Ang2 than with autocrine ANG2 in transgenic mice could depend on differences in ANG2 concentration and site or duration 
of expression (61). Furthermore, the agonist activity of recombinant ANG2 can be increased by tetramer formation or the chimeric domains used to increase ANG2 solubility and stability $(70,71)$.

LPS triggers ANG2 release and decreases ANG1-Tie2/Tie1 signaling. Recombinant ANG1, inhibition of ANG2 or increasing Tie2 activity by therapeutic antibodies or a small-molecule phosphatase inhibitor have vascular protective effects in acute inflammation and in sepsis $(32,33,34,62,72,73)$, consistent with an agonist action of ANG1 and antagonistic action of ANG2. Reduced Tie2 protein and Tie2 and Ang1 mRNA levels have been reported in murine sepsis and systemic inflammation $(56,74)$. We confirmed these findings and additionally found reduced Tie1 mRNA in mice 3 to 12 hours after administration of endotoxin (LPS), a potent microbial mediator of the pathogenesis of Gram-negative bacterial sepsis and septic shock. We found that LPS induced the release of preformed ANG2 from Weibel-Palade bodies in endothelial cells of tracheal vessels within 30 minutes and promoted FOXO1 translocation to nucleus within 6 hours, which provides a positive feedback loop for Ang2 gene transcription (75). These rapid events are likely to contribute to the subsequent increase in Ang2 mRNA expression in the lungs and ANG2 protein in serum as well as to the reduction of Tie2 phosphorylation.

Rapid cleavage of Tie1 and loss of ANG2 agonist activity in inflammation. VEGF and inflammatory stimuli, including TNF- $\alpha$, can lead to Tie1 ectodomain cleavage in endothelial cells $(60,76)$, and sTie1 can be detected in human serum (77). We found that in acute inflammation, endothelial Tie1 was rapidly cleaved (within 30 minutes of LPS application), resulting in loss of the Tie1 ectodomain that mediates angiopoietin-induced Tie1-Tie2 interaction. LPS-induced Tie1 ectodomain shedding correlated with loss of autocrine ANG2-induced Tie2 phosphorylation in Ang $2^{E C}$ mice. This finding fits with our results demonstrating that genetic Tie1 deletion impairs the Tie2 agonist activity of angiopoietins. However, it should be noted that Tie1 deletion differs from LPS-mediated Tie1 cleavage, which results in shedding of the sTie1 and production of the Tie1 intracellular domain with unknown functions in inflammation. These results also suggest that in inflammation Tie1 cleavage abrogates the agonist activity of autocrine ANG2 released from the Weibel-Palade bodies. As a result, decreased Tie2 signaling relieves the tonic suppression of FOXO1 activity, leading to increased expression of ANG2 and vessel destabilization typical of the leaky vessels in inflammation. Interestingly, Ad-CAng1 pretreatment reduced LPS-induced Tie1 cleavage, increase in ANG2, and vascular leakage. The partial rescue of Tie1 levels could contribute to the vascular stabilizing functions of ANG1. These results suggest that inflammatory signals promote the function of Ang2 as an antagonist through Tie1 ectodomain shedding. Similar results were obtained by Kim et al. (70).

Additional advances in understanding the actions of Tie 1 will come from elucidation of the mechanistic differences between the acute inflammation induced by LPS, characterized by rapid Tie1 ectodomain cleavage, and the more gradual onset of chronic inflammatory conditions and atherosclerosis. Tie1 expression is elevated at sites of nonlaminar shear stress in the arterial vessel wall, where atheromas develop; partial genetic deletion of Tie1 protects from the development of atherosclerosis (25). Inflammation leads to vessel enlargement with decreased laminar shear stress. In these conditions, increased Tie1 expression could promote inflammatory responses $(24,25)$. However, the rapid kinetics of Tie1 cleavage complicates the comparisons of acute and chronic inflammation models. In the absence of genetic and pharmacological tools to manipulate Tie1 cleavage, the full significance of ectodomain shedding in LPS-induced inflammation and leakage remains to be determined. Future work should elucidate the respective contributions of the cleaved Tie1 ectodomain and the intracellular tyrosine kinase domain in acute inflammation.

Of clinical relevance, we detected significantly higher concentrations of sTie1 ectodomain in the serum of patients with acute PUUV infection $(P<0.001)$, which justifies further studies of sTie1 in human inflammatory conditions. Reduced Tie1 RNA is linked to susceptibility to Ebola virus-induced hemorrhagic fever and vascular leakage in mice (78), providing evidence that loss of Tie1 is associated with disease pathogenesis and contributes to reduced vascular stability.

In summary, our results define Tie 1 as an essential component of angiopoietin/Tie2 receptor interaction, signaling, and trafficking. Tie1 is also essential for the agonist action of ANG1 and ANG2 on Tie2 during vascular remodeling in noninflammatory conditions. In addition, the findings suggest that through ectodomain cleavage, Tie1 is a molecular switch that suppresses or blocks the agonist activity of ANG1 and ANG2 on Tie2. Together, the results point to Tie1 as a target for therapeutic intervention in acute inflammation.

\section{Methods}

Additional details are available in the Supplemental Methods.

Mouse models. The Tie $1^{f / f l}$ (20), Tie ${ }^{\text {LacZ/+ }}$ (15), Pdgfb-iCreER ${ }^{T 2}$ (gift from Marcus Fruttiger, Institute of Ophthalmology, University College London, London, United Kingdom) (79), Cdh5(PAC)-CreER ${ }^{T 2}$ (gift from Ralf Adams, Max Planck Institute for Molecular Biomedicine, Münster, Germany) (80), VE-cadherin-tTA (Cdh5-tTA) (gift from Laura Benjamin, Beth Israel Deaconess Medical Center, Harvard Medical School, Boston, Massachusetts, USA) (81), Tie1-tTA (gift from Daniel Dumont, Sunnybrook Research Institute, Toronto, Canada) (82), and tetO-Ang2 (50) mouse lines have been published previously. Endothelial-specific deleters (Pdgfb-iCreER $R^{T 2}$ and Cdh5[PAC]-CreER ${ }^{T 2}$ ) were used for inducible inactivation of Tie1 by daily administration of tamoxifen by oral gavage ( $2 \mathrm{mg} / \mathrm{mouse} / \mathrm{d}$ ) for 5 days in 6- to 10-week-old Pdgfb-iCreER ${ }^{T 2}$ Tie $1^{f / / f l}, \quad P d g f b-i C r e E R^{T 2} T i e 1^{f / L a c Z}, C d h 5(P A C)-C r e E R^{T 2} T i e 1^{f / / f l}$, and Cdh5(PAC)-CreERT2 Tie $1^{f / L a c Z}$ mice. Cre-positive Tie $1^{f / f l}$ and Tie $1^{\mathrm{LacZ} / f l}$ mice were compared with Cre-negative Tie $1^{f / f l}$ and Tie $1^{\text {LacZ/fl }}$ littermates. Both Cre-negative and Cre-positive Tie1 targeted mice were similarly treated with tamoxifen. We observed reduced vascular remodeling and endothelial cell proliferation with both Cre deleters. We have observed that Tie1 deletions resulting in less than $90 \%$ reduction in Tie1 protein levels do not show a vascular phenotype in postnatal retinal angiogenesis (20); therefore we were able to use Tie $1^{f / f l}$ and Tie $1^{\text {LacZ/fl }}$ littermates as phenotypically WT controls. Thus, control was used to refer to the Cre-negative Tie $1^{f / f l}$ and Tie $1^{\text {LacZ/fl }}$ littermates. Cre-positive Tie $1^{f / f l}$ and Tie $1^{\text {LacZ/fl }}$ mice were referred to as Tie ${ }^{i \text { SEC }}$. None of the mice had 2 Cre alleles. The Cre deleter strains used are indicated in the figure legends.

To obtain double-transgenic ANG2-overexpressing mice, the driver (Cdh5-tTA [ref. 81] or Tie1-tTA [ref. 82]) and responder (tetOAng2 [ref. 50]) transgenic mouse lines were bred together. Cdh5-tTA 
mice were used in the C57BL/6JCrl background and Tie1-tTA in the FVB background. Single transgenic or WT littermates were used as controls. ANG2 expression was repressed until birth by administration of $2 \mathrm{mg} / \mathrm{ml}$ of tetracycline in $5 \%$ sucrose in the drinking water of pregnant females. The levels of serum ANG2 were about $20 \mathrm{ng} /$ $\mathrm{ml}$ in WT mice and $500 \mathrm{ng} / \mathrm{ml}$ in the ANG2 transgenic mice induced for 2 to 3 months after birth. To delete Tie1 in $A n g 2^{E C}$ mice, tamoxifen was administered 3 times per week for a total of 6 to 8 times to Pdgfb-iCreER $R^{T 2}$ Tie $^{\text {flflfl }}$ Cdh5-tTA tetO-Ang2 mice and to their controls. Both male and female mice were used, all mice used were in the C57BL/6JCrl background unless indicated otherwise, and all experiments had littermate controls. WT mice were ordered from Scanbur (C57BL/6JCrl) or Harlan (C57BL/6J OlaHsd).

Adenoviral gene transfer. $1 \times 10^{9} \mathrm{PFUs} /$ mouse of empty control or LacZ (Ad-control), COMP-Ang1 (Ad-CAng1), or mANG2 (Ad-Ang2) adenovirus vector in $100 \mu \mathrm{l}$ of PBS were injected into control and Tie1-deficient mice via tail vein (26). Two days or 2 weeks later, the mice were sacrificed and their tracheas, lungs, and serum were collected for analysis.

LPS treatment. Endotoxemia was induced in mice by i.p. injection of LPS (15 mg/kg of body weight, E. coli LPS 055:B5, Sigma-Aldrich). Mice were sacrificed at $0.5,1,3,6,12$, or 16 hours after LPS challenge. Tie2 phosphorylation and Tie1 levels were analyzed in lung lysates from 20- to 35-week-old control and $A n g 2^{E C}$ mice or 11- to 14-weekold Ad-control- or Ad-CAng1-treated mice with or without LPS (16 hours). Adenoviruses were injected 2 days prior to LPS treatment.

Cell culture and stimulations. HUVECs (PromoCell) were grown in Endothelial Cell Basal Medium MV (ECBM) supplemented with Supplement Pack GM MV (PromoCell) on gelatin-coated (0.1\%) culture plates or coverslips. Confluent HUVECs were starved for 3 to 5 hours in $1 \%$ FCS containing ECBM. Starved HUVECs were treated for 10 to 30 minutes with CAng1 (49) (200-500 ng/ml), recombinant human ANG1 (R\&D Systems, 100-500 ng/ml), or recombinant human ANG2 (R\&D Systems, 200-500 ng/ml) or VEGF (R\&D Systems, 50 ng/ml). HUVECs were fixed with $4 \%$ paraformaldehyde (PFA) for 10 minutes at room temperature (RT), washed 3 times with PBS for 5 minutes, and subjected to immunofluorescence staining.

HUVECs were transduced at $50 \%$ confluence by using retroviral vector-containing cell supernatants and polybrene (0.1\%, SigmaAldrich) and analyzed after 48 hours. For integrin silencing using shRNA lentiviral vectors, 50\%-90\% confluent HUVECs were transduced with integrin or Scr shRNA lentivirus particles in the presence of $0.1 \%$ polybrene for 5 hours, after which the ECBM medium was added. Fresh medium was exchanged after overnight incubation, and the cells were analyzed after 48 hours. For analysis of FOXO1 and cell proliferation, HUVECs were transfected (for 24 hours) with lentivirus encoding preselected $\beta_{1}$ integrin or Tie1 shRNA clones or shScr and thereafter incubated in the presence of puromycin for 2 days. For analysis of mitoses, HUVECs treated as indicated above were cultured in the Cell-IQ Live Cell Imaging System. Images were acquired every 20 minutes, and mitoses were counted manually. Additionally, the number of live cells (indicative of ongoing cell proliferation) was determined using the MTT assay.

Physiological and biochemical analysis. All experiments were done according to protocols described in the Supplemental Methods.

FRET and FLIM measurements. FRET with acceptor photobleaching was performed using a Leica TCS SP2 confocal microscope with
$63 \times$ HCX PL APO CS oil-immersion objective NA 1.40 or a Leica TCS SP8 confocal microscope with $63 \times$ HC PL APO CS2 oil-immersion objective NA 1.30, using an excitation wavelength of $488 \mathrm{~nm}$ (argon laser) for GFP and of $561 \mathrm{~nm}$ (DPSS laser) for Cy3. Fluorescence emissions at 500-530 nm and 570-632 $\mathrm{nm}$ were recorded for GFP and Cy3, respectively. For acceptor photobleaching, regions of interest (ROI) were bleached at $100 \%$ of DPSS laser power as long as the Cy3 fluorescence intensity decreased to $0 \%-15 \%$, as monitored by the Leica software. Pre- and postbleach images were recorded for both donor (GFP) and acceptor (Cy3). FRET efficiency was calculated as follows: FRET efficiency $=($ Dpost - Dpre $) / D$ post , where Dpre and Dpost are the donor intensity before and after photobleaching, respectively. Photobleached GFP images were verified by comparing GFP intensity outside the bleached area before and after bleaching. Cells that displayed a drift in the $x-y$ focal plane were discarded before FRET analysis. FRET efficiencies were analyzed using ImageJ software (NIH). To obtain homogenous levels of receptor expression, retroviral transfected HUVECs used for FRET/FLIM analysis were sorted for Tie1 and Tie2 expression by FACS.

Frequency domain FLIM was performed using 3I Marianas (3I intelligent Imaging Innovations) fluorescence microscope with $63 \times$ Alpha Plan-Apochromat oil-immersion objective NA 1.46 and $488 \mathrm{~nm}$ solid-state laser modulated at $50 \mathrm{MHz}$. The donor was excited using Semrock LF488-B filter set. Images were processed using Slidebook 5.5, and GFP lifetimes were calculated from ROI. More information about the method can be found elsewhere (83).

Confocal FLIM based on TCSPC was performed using Zeiss LSM 880 confocal microscope equipped with FRET module with 63× Plan-Apochromat oil-immersion objective NA 1.40 and PDL 800-D Diode laser driver for picosecond pulses, modulated at 40 MHz. GFP was excited with $485 \pm 10 \mathrm{~nm}$ (LDH-D Series, PicoQuant). Images of $256 \times 256$ pixels were acquired detecting $6 \times$ $10^{4}-10^{5}$ photons. Images of the donor fluorescence were processed using SymPhoTime64 software, and GFP lifetimes were calculated from ROI after fitting to GFP lifetime decay functions. Images of the donor fluorescence are presented in pseudocolor photon counting setup (PicoQuant).

Patient samples. The study cohort consisted of serum from 23 PUUV-infected patients and 10 controls. The PUUV-infected patient samples have been described recently (84). The median age of patients was 38 years (range 22-69) and the median age of controls 37 years (range 21-52). The patient cohort consisted of samples from 19 men and 4 women. Control samples were from 3 men and 7 women. sTie1 from patient serum samples was analyzed by hTie1 ELISA (R\&D).

Statistics. Results are expressed as mean \pm SEM or \pm SD. Statistical analysis was carried out using Student's $t$ 2-tailed test or 1-way ANOVA, followed by Tukey's, Dunnett's, Bonferroni's or Fisher's least significant difference (LSD) test with SPSS or Prism Statistical analysis. Welch's $t$ test (2 tailed, unequal variance) was used for pairwise comparisons of FLIM and FRET measurements followed by Bonferroni's test. A $P$ value of less than 0.05 was considered to be statistically significant.

Study approval. The National Animal Experiment Board in Finland approved all experiments involving the use of mice. Mice were housed in individually ventilated cages with enrichment materials in a facility following the guidelines by the Federation of European Laboratory Animal Science Associations. Patient samples 
were obtained from a study approved by the Ethics Committee of the Tampere University Hospital. Written informed consent was obtained from all patients.

\section{Author contributions}

EAK and AL designed and conducted experiments, analyzed and interpreted results, and contributed to the writing of the manuscript. HG, AA, MK, BA, SF, GD, and ML designed and conducted experiments and analyzed data. TS, AV, TJS, SYH, and GYK provided materials. DMM contributed to the design of experiments. PS and KA designed the study, interpreted the results, and wrote the manuscript.

\section{Acknowledgments}

We thank Tapio Tainola, Riitta Kauppinen, Laura Raitainen, and Kirsi Mänttäri for technical assistance, the Functional Genomics Unit for TRC1 library clones, Jussi Kenkkilä and the Biomedicum Imaging Unit for microscopy services and assistance with TCSPC, the Laboratory Animal Center of the University of Helsinki for technical assistance, Anthony Squire for assistance with FRET analysis, and Marko Crivaro and Kimmo Tanhuanpää at the
Light Microscopy Unit, University of Helsinki, for assistance with the FLIM analysis. We also thank Laura Benjamin for the Cdh5tTA mouse line, Ralf Adams for the Cdh5(PAC)-CreER ${ }^{T 2}$ mice, Daniel Dumont for the Tie1-tTA mice, Marcus Fruttiger for the Pdgfb-iCreER ${ }^{T 2}$ mice, and Elisabetta Dejana for the VE-cadherin cDNA. This work was funded by the Academy of Finland (Centre of Excellence Program 2014-2019 (271845 to PS and KA), an European Research Council (ERC) Advanced Grant (ERC-2010AdG-268804 to KA), the Leducq Foundation (11CVD03 to KA and DMM), the Marie Curie Actions of European Union Seventh Framework Programme (FP7/2007-2013 grant 317250), the Sigrid Juselius Foundation (to PS and KA), the Finnish Cultural Foundation (to PS and AL), the Finnish Cancer Organizations (to PS, KA, and EAK), the National Heart, Lung, and Blood Institute (P01 HL024136, R01 HL059157, and R01 HL127402) of the NIH (to DMM), the Biomedicum Helsinki Foundation (to EAK) and the Ida Montin Foundation (to EAK and AL).

Address correspondence to: Kari Alitalo, Biomedicum Helsinki, PO Box 63 (Haartmaninkatu 8), FI-00014 University of Helsinki, Finland. Phone: 358.2.941.25511; E-mail: kari.alitalo@helsinki.fi.
1. $\mathrm{Hu} \mathrm{J}$, et al. Endothelial cell-derived angiopoietin-2 controls liver regeneration as a spatiotemporal rheostat. Science. 2014;343(6169):416-419.

2. Ramasamy SK, Kusumbe AP, Adams RH. Regulation of tissue morphogenesis by endothelial cell-derived signals. Trends Cell Biol. 2015;25(3):148-157.

3. Jeltsch M, Leppänen VM, Saharinen P, Alitalo K. Receptor tyrosine kinase-mediated angiogenesis. Cold Spring Harb Perspect Biol. 2013;5(9):a009183.

4. Augustin HG, Koh GY, Thurston G, Alitalo K. Control of vascular morphogenesis and homeostasis through the angiopoietin-Tie system. Nat Rev Mol Cell Biol. 2009;10(3):165-177.

5. Saharinen P, Jeltsch M, Santoyo MM, Leppänen VM, Alitalo K. The TIE Receptor Family. In: Wheeler DE, Yarden Y, eds. Receptor Tyrosine Kinases: Family and Subfamilies. Springer International Publishing Switzerland; 2015:743-775.

6. Partanen J, et al. A novel endothelial cell surface receptor tyrosine kinase with extracellular epidermal growth factor homology domains. $\mathrm{Mol}$ Cell Biol. 1992;12(4):1698-1707.

7. Dumont DJ, Yamaguchi TP, Conlon RA, Rossant J, Breitman ML. tek, a novel tyrosine kinase gene located on mouse chromosome 4 , is expressed in endothelial cells and their presumptive precursors. Oncogene. 1992; 7(8):1471-1480.

8. Davis S, et al. Isolation of angiopoietin-1, a ligand for the TIE2 receptor, by secretion-trap expression cloning. Cell. 1996;87(7):1161-1169.

9. Maisonpierre PC, et al. Angiopoietin-2, a natural antagonist for Tie2 that disrupts in vivo angiogenesis. Science. 1997;277(5322):55-60.

10. Daly C, et al. Angiopoietin-1 modulates endothelial cell function and gene expression via the transcription factor FKHR (FOXO1). Genes Dev. 2004;18(9):1060-1071.

11. Zhang X, et al. Phosphorylation of serine 256 suppresses transactivation by FKHR (FOXO1) by multiple mechanisms. Direct and indirect effects on nuclear/cytoplasmic shuttling and DNA binding. J Biol Chem. 2002;277(47):45276-45284.

12. Wilhelm K, et al. FOXO1 couples metabolic activity and growth state in the vascular endothelium. Nature. 2016;529(7585):216-220.

13. Puri MC, Partanen J, Rossant J, Bernstein A. Interaction of the TEK and TIE receptor tyrosine kinases during cardiovascular development. Development. 1999;126(20):4569-4580.

14. Jeansson M, et al. Angiopoietin-1 is essential in mouse vasculature during development and in response to injury. JClin Invest. 2011;121(6):2278-2289.

15. Puri MC, Rossant J, Alitalo K, Bernstein A, Partanen J. The receptor tyrosine kinase TIE is required for integrity and survival of vascular endothelial cells. EMBO J. 1995;14(23):5884-5891.

16. D’Amico G, Korhonen EA, Waltari M, Saharinen P, Laakkonen P, Alitalo K. Loss of endothelial Tie1 receptor impairs lymphatic vessel development-brief report. Arterioscler Thromb Vasc Biol. 2010;30(2):207-209.

17. Qu X, Tompkins K, Batts LE, Puri M, Baldwin HS Abnormal embryonic lymphatic vessel development in Tie1 hypomorphic mice. Development. 2010;137(8):1285-1295.

18. Shen B, et al. Genetic dissection of tie pathway in mouse lymphatic maturation and valve development. Arterioscler Thromb Vasc Biol. 2014;34(6):1221-1230.

19. Qu X, Zhou B, Scott Baldwin H. Tie1 is required for lymphatic valve and collecting vessel development. Dev Biol. 2015;399(1):117-128.

20. D’Amico G, et al. Tie1 deletion inhibits tumor growth and improves angiopoietin antagonist therapy. J Clin Invest. 2014;124(2):824-834.

21. Savant S, et al. The Orphan Receptor Tie1 Controls Angiogenesis and Vascular Remodeling by Differentially Regulating Tie2 in Tip and Stalk Cells. Cell Rep. 2015;12(11):1761-1773.

22. Korhonen J, et al. Enhanced expression of the tie receptor tyrosine kinase in endothelial cells during neovascularization. Blood. 1992;80(10):2548-2555.

23. Kaipainen A, et al. Enhanced expression of the tie receptor tyrosine kinase mesenger RNA in the vascular endothelium of metastatic melanomas. Cancer Res. 1994;54(24):6571-6577.

24. Chan B, Sukhatme VP. Suppression of Tie-1 in endothelial cells in vitro induces a change in the genome-wide expression profile reflecting an inflammatory function. FEBS Lett. 2009;583(6):1023-1028.

25 . Woo KV, et al. Tie1 attenuation reduces murine atherosclerosis in a dose-dependent and shear stress-specific manner. J Clin Invest. 2011;121(4):1624-1635.

26. Kim KE, Cho CH, Kim HZ, Baluk P, McDonald $\mathrm{DM}, \mathrm{Koh} \mathrm{GY}$. In vivo actions of angiopoietins on quiescent and remodeling blood and lymphatic vessels in mouse airways and skin. Arterioscler Thromb Vasc Biol. 2007;27(3):564-570.

27. Fuxe J, et al. Angiopoietin/Tie2 signaling transforms capillaries into venules primed for leukocyte trafficking in airway inflammation. $\mathrm{Am} J$ Pathol. 2010;176(4):2009-2018.

28. Tabruyn SP, et al. Angiopoietin-2-driven vascular remodeling in airway inflammation. Am J Pathol. 2010;177(6):3233-3243.

29. Le CT, et al. Synergistic actions of blocking angiopoietin- 2 and tumor necrosis factor- $\alpha$ in suppressing remodeling of blood vessels and lymphatics in airway inflammation. Am J Pathol. 2015;185(11):2949-2968.

30. Parikh SM, et al. Excess circulating angiopoietin-2 may contribute to pulmonary vascular leak in sepsis in humans. PLoS Med. 2006;3(3):e46.

31. David S, et al. Angiopoietin-2 may contribute to multiple organ dysfunction and death in sepsis*. Crit Care Med.2012;40(11):3034-3041.

32. Ziegler T, et al. Angiopoietin 2 mediates microvascular hemodynamic alterations in sepsis. 
JClin Invest. 2013;123(8):3436-3445.

33. David S, et al. Acute administration of recombinant Angiopoietin-1 ameliorates multiple-organ dysfunction syndrome and improves survival in murine sepsis. Cytokine. 2011;55(2):251-259.

34. McCarter SD, et al. Cell-based angiopoietin-1 gene therapy for acute lung injury. Am J Respir Crit Care Med. 2007;175(10):1014-1026.

35. Ghosh CC, et al. Gene control of tyrosine kinase TIE2 and vascular manifestations of infections. Proc Natl Acad Sci U S A. 2016;113(9):2472-2477.

36. Syrjälä SO, et al. Angiopoietin-2 inhibition prevents transplant ischemia-reperfusion injury and chronic rejection in rat cardiac allografts. Am J Transplant. 2014;14(5):1096-1108.

37. Hammes HP, et al. Angiopoietin- 2 causes pericyte dropout in the normal retina: evidence for involvement in diabetic retinopathy. Diabetes. 2004;53(4):1104-1110.

38. Felcht $\mathrm{M}$, et al. Angiopoietin-2 differentially regulates angiogenesis through TIE2 and integrin signaling. J Clin Invest. 2012;122(6):1991-2005.

39. Park SW, Yun JH, Kim JH, Kim KW, Cho CH, Kim $\mathrm{JH}$. Angiopoietin 2 induces pericyte apoptosis via a3ß1 integrin signaling in diabetic retinopathy. Diabetes. 2014;63(9):3057-3068.

40. Hakanpaa L, et al. Endothelial destabilization by angiopoietin- 2 via integrin $\beta 1$ activation. $N a t$ Commun. 2015;6:5962.

41. Cascone I, Napione L, Maniero F, Serini G, Bussolino F. Stable interaction between $\alpha 5 \beta 1$ integrin and Tie2 tyrosine kinase receptor regulates endothelial cell response to Ang-1. J Cell Biol. 2005;170(6):993-1004.

42. Saharinen $P$, et al. Multiple angiopoietin recombinant proteins activate the Tie1 receptor tyrosine kinase and promote its interaction with Tie2. JCell Biol. 2005;169(2):239-243.

43. Yuan HT, et al. Activation of the orphan endothelial receptor Tie1 modifies Tie2-mediated intracellular signaling and cell survival. FASEB J. 2007;21(12):3171-3183.

44. Fukuhara S, et al. Differential function of Tie2 at cell-cell contacts and cell-substratum contacts regulated by angiopoietin-1. Nat Cell Biol. 2008;10(5):513-526.

45. Saharinen P, et al. Angiopoietins assemble distinct Tie2 signalling complexes in endothelial cell-cell and cell-matrix contacts. Nat Cell Biol. 2008;10(5):527-537.

46. Kontos CD, Cha EH, York JD, Peters KG. The endothelial receptor tyrosine kinase Tie1 activates phosphatidylinositol 3-kinase and Akt to inhibit apoptosis. Mol Cell Biol. 2002;22(6):1704-1713.

47. Marron MB, et al. Regulated proteolytic processing of Tie1 modulates ligand responsiveness of the receptor-tyrosine kinase Tie2.J Biol Chem. 2007;282(42):30509-30517.

48. Seegar TC, et al. Tie1-Tie2 interactions mediate functional differences between angiopoietin ligands. Mol Cell. 2010;37(5):643-655.

49. Cho $\mathrm{CH}$, et al. COMP-Ang1: a designed angiopoietin-1 variant with nonleaky angiogenic activity. Proc Natl Acad Sci U S A. 2004;101(15):5547-5552.

50. Holopainen T, et al. Effects of angiopoietin-2-blocking antibody on endothelial cell-cell junctions and lung metastasis. J Natl Cancer Inst. 2012;104(6):461-475

51. Kim M, Carman CV, Springer TA. Bidirectional transmembrane signaling by cytoplasmic domain separation in integrins. Science. 2003;301(5640):1720-1725.

52. Haj FG, Verveer PJ, Squire A, Neel BG, Bastiaens PI. Imaging sites of receptor dephosphorylation by PTP1B on the surface of the endoplasmic reticulum. Science. 2002;295(5560):1708-1711.

53. Grecco HE, et al. In situ analysis of tyrosine phosphorylation networks by FLIM on cell arrays. Nat Methods. 2010;7(6):467-472.

54. Daly C, et al. Angiopoietin-2 functions as an autocrine protective factor in stressed endothelial cells. Proc Natl Acad Sci U S A. 2006;103(42):15491-15496.

55. Cho $\mathrm{CH}$, et al. Long-term and sustained COMP-Ang1 induces long-lasting vascular enlargement and enhanced blood flow. Circ Res. 2005;97(1):86-94.

56. Mofarrahi M, Nouh T, Qureshi S, Guillot L, Mayaki D, Hussain SN. Regulation of angiopoietin expression by bacterial lipopolysaccharide. Am J Physiol Lung Cell Mol Physiol. 2008;294(5):L955-L963.

57. Scholz A, Plate KH, Reiss Y. Angiopoietin-2: a multifaceted cytokine that functions in both angiogenesis and inflammation. Ann N Y Acad Sci. 2015;1347:45-51.

58. Vaheri A, et al. Uncovering the mysteries of hantavirus infections. Nat Rev Microbiol. 2013;11(8):539-550.

59. Tisoncik JR, Korth MJ, Simmons CP, Farrar J, Martin TR, Katze MG. Into the eye of the cytokine storm. Microbiol Mol Biol Rev. 2012;76(1):16-32.

60. Singh H, Hansen TM, Patel N, Brindle NP. The molecular balance between receptor tyrosine kinases Tie1 and Tie2 is dynamically controlled by VEGF and TNF $\alpha$ and regulates angiopoietin signalling. PLoS ONE. 2012;7(1):e29319.

61. Teichert-Kuliszewska K, et al. Biological action of angiopoietin-2 in a fibrin matrix model of angiogenesis is associated with activation of Tie2. Cardiovasc Res. 2001;49(3):659-670.

62. Han S, et al. Amelioration of sepsis by TIE2 activation-induced vascular protection. Sci Transl Med.2016;8(335):335ra55.

63. Gale NW, et al. Angiopoietin-2 is required for postnatal angiogenesis and lymphatic patterning, and only the latter role is rescued by Angiopoietin-1. Dev Cell. 2002;3(3):411-423.

64. Lee J, et al. Angiopoietin-1 guides directional angiogenesis through integrin $\alpha v \beta 5$ signaling for recovery of ischemic retinopathy. Sci Transl Med. 2013;5(203):203ra127.

65. Patan S. TIE1 and TIE2 receptor tyrosine kinases inversely regulate embryonic angiogenesis by the mechanism of intussusceptive microvascular growth. Microvasc Res. 1998;56(1):1-21.

66. Thomson BR, et al. A lymphatic defect causes ocular hypertension and glaucoma in mice. J Clin Invest. 2014;124(10):4320-4324.

67. Nätynki M, et al. Common and specific effects of Tie2 mutations causing venous malformations. Hum Mol Genet. 2015;24(22):6374-6389.
68. Boscolo E, et al. Rapamycin improves TIE2mutated venous malformation in murine model and human subjects. JClin Invest. 2015;125(9):3491-3504.

69. Yuan HT, Khankin EV, Karumanchi SA, Parikh SM. Angiopoietin 2 is a partial agonist/antagonist of Tie2 signaling in the endothelium. Mol Cell Biol. 2009;29(8):2011-2022.

70. Kim M, et al. Opposing actions of angiopoietin-2 on Tie2 signaling and FOXO1 activation. J Clin Invest.2016;126(9):3511-3525.

71. Kim HZ, Jung K, Kim HM, Cheng Y, Koh GY. A designed angiopoietin-2 variant, pentameric COMP-Ang2, strongly activates Tie2 receptor and stimulates angiogenesis. Biochim Biophys Acta. 2009;1793(5):772-780.

72. Frye M, et al. Interfering with VE-PTP stabilizes endothelial junctions in vivo via Tie-2 in the absence of VE-cadherin. JExp Med. 2015;212(13):2267-2287.

73. Stiehl T, et al. Lung-targeted RNA interference against angiopoietin-2 ameliorates multiple organ dysfunction and death in sepsis. Crit Care Med. 2014;42(10):e654-662.

74. Kurniati NF, et al. The flow dependency of Tie2 expression in endotoxemia. Intensive Care Med. 2013;39(7):1262-1271.

75. Ghosh CC, et al. Drug Repurposing Screen Identifies Foxo1-Dependent Angiopoietin-2 Regulation in Sepsis. Crit Care Med. 2015;43(7):e230-e240.

76. Yabkowitz R, Meyer S, Black T, Elliott G, Merewether LA, Yamane HK. Inflammatory cytokines and vascular endothelial growth factor stimulate the release of soluble tie receptor from human endothelial cells via metalloprotease activation. Blood.1999;93(6):1969-1979.

77. Karnani P, Kairemo K. The new Tie-1 monoclonal antibodies detect angiogenesis in metastatic malignancies. Clin Cancer Res. 2003; 9(10 Pt 2):3827S-3830S.

78. Rasmussen AL, et al. Host genetic diversity enables Ebola hemorrhagic fever pathogenesis and resistance. Science. 2014;346(6212):987-991.

79. Claxton S, Kostourou V, Jadeja S, Chambon P, Hodivala-Dilke K, Fruttiger M. Efficient, inducible Cre-recombinase activation in vascular endothelium. Genesis. 2008;46(2):74-80.

80. Wang Y, et al. Ephrin-B2 controls VEGF-induced angiogenesis and lymphangiogenesis. Nature. 2010;465(7297):483-486.

81. Sun JF, et al. Microvascular patterning is controlled by fine-tuning the Akt signal. Proc Natl Acad Sci U S A. 2005;102(1):128-133.

82. Sarao R, Dumont DJ. Conditional transgene expression in endothelial cells. Transgenic Res. 1998;7(6):421-427.

83. Verveer PJ, Rocks O, Harpur AG, Bastiaens PI. Imaging protein interactions by FRET microscopy: FLIM measurements. CSH Protoc. 2006;2006(6):pdb.prot4599.

84. Strandin T, et al. Interferons Induce STAT1Dependent Expression of Tissue Plasminogen Activator, a Pathogenicity Factor in Puumala Hantavirus Disease. J Infect Dis. 2016;213(10):1632-1641. 